\title{
Total Synthesis of (-)-Colchicine via a Rh-triggered Cycloaddition Cascade
}

\author{
Timm Graening, Virginie Bette, Jörg Neudörfl, Johann Lex and Hans-Günther Schmalz* \\ Institut für Organische Chemie, Universität zu Köln, Greinstr. 4, D-50939 Köln, Germany \\ schmalz@uni-koeln.de
}

\section{Supporting Information}

\begin{tabular}{|c|c|c|c|}
\hline General Procedures & 2 & Tropolones 21 and 22 & 17 \\
\hline \multicolumn{4}{|c|}{ Tropolonemethyl ethers $\mathbf{2 4}$ and $\mathbf{2 5}$} \\
\hline & & 7-Hydroxydesacetamidocolchicine $\mathbf{2 6}$ & 20 \\
\hline Propargylic ketone 6 & 2 & 7-Hydroxydesacetamido- & \\
\hline Propargylic alcohol 8 & 3 & -isocolchicine 27 & 21 \\
\hline Silyl ether 9 & 4 & Methanesulfonic acid $\mathbf{2 8}$ & 22 \\
\hline 4-oxobutyric acid $\mathbf{1 0}$ & 5 & Azide 30 & 23 \\
\hline Diazoketone 11 & 6 & Colchicine 1 & 24 \\
\hline TBS-protected Cycloadduct 12 & 7 & & \\
\hline Cycloadduct 13 & 8 & Crystallographic Data & \\
\hline Tropone 14 & 10 & Compound 8 & 25 \\
\hline oxa-bridged compound $\mathbf{1 5}$ & 11 & Compound 12 & 27 \\
\hline Diol 16 & 12 & Compound 25 & 28 \\
\hline Aminotropones $\mathbf{1 7}$ and $\mathbf{1 8}$ & 13 & Compound 32 & 29 \\
\hline Aminotropones 19 and 20 & 15 & & \\
\hline
\end{tabular}


General Procedures. Reactions were conducted in flame-dried glassware under an atmosphere of argon using freshly distilled anhydrous solvents. NMR spectra were recorded at $25{ }^{\circ} \mathrm{C}$ on Bruker DRX 500, Bruker DPX 300 or Bruker AC 250 spectrometers. Proton chemical shifts are reported in ppm $(\delta)$ relative to the solvent reference downfield from TMS and were determined by reference to the residual solvent peaks $\left(\mathrm{CDCl}_{3}: \delta 7.24 \mathrm{ppm}\right.$, DMSO [d6]: $2.50 \mathrm{ppm}$ ). Data are reported as follows: chemical shift (multiplicity [singlet (s), doublet (d), triplet (t), quartet (q) and multiplet (m)], coupling constants [Hz], integration). Carbon NMR spectra were recorded with complete proton decoupling and the multiplicity was assessed by DEPT measurements. Carbon chemical shifts are reported in ppm $(\delta)$ relative to solvent resonance as the internal standard $\left(\mathrm{CDCl}_{3}: \delta 77.0 \mathrm{ppm}, \mathrm{DMSO}[\mathrm{d} 6]: 39.5 \mathrm{ppm}\right)$. Infrared spectra were obtained on Perkin Elmer FT-IR Paragon 1000 spectrometer. Melting points were recorded on a Büchi B-545 and are not corrected. Optical rotations were recorded on a Perkin Elmer Polarimeter 343 plus at the given wavelengths (path length $100 \mathrm{~mm}$ ). Mass spectra were obtained on Finnigan MAT Incos 50 Galaxy System (DIP-MS) (EI) or Finnigan MAT 900 (ESI) spectrometers, high resolution mass spectra on a Finnigan HSQ-30 (HR-EIMS) or on a Finnigan MAT 900 (HR-ESI-MS). The method of ionisation is given in parentheses.

\section{Characterizations}

\section{5-(2-Iodo-3,4,5-trimethoxyphenyl)-1-(trimethylsilyl)pent-1-yn-3-one (6)}

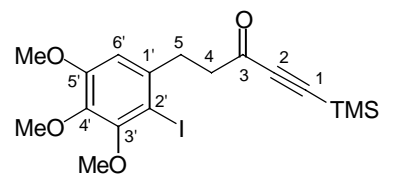

To a solution of TMS-acetylene (15.5 mL, $112 \mathrm{mmol}, 1.53$ equiv.) in abs. THF (215 mL) was slowly added a solution of $n$-BuLi (68.1 mL, $106 \mathrm{mmol}, 1.45$ equiv., $1.56 \mathrm{M}$ in hexane) under argon at $-78^{\circ} \mathrm{C}$. After $30 \mathrm{~min}$, the resulting solution was warmed to $0{ }^{\circ} \mathrm{C}$ and cooled then back to $-78^{\circ} \mathrm{C}$. The lithiumacetylid-solution obtained was added dropwise through a canula to a solution of Weinreb amide $(7)(30.0 \mathrm{~g}, 73.3 \mathrm{mmol})$ in abs. THF $(215 \mathrm{~mL})$ at $-78^{\circ} \mathrm{C}$. The resulting solution was warmed to $-10^{\circ} \mathrm{C}$ over $1 \mathrm{~h}$, after $60 \mathrm{~min}$ cooled to $-40{ }^{\circ} \mathrm{C}$ and then

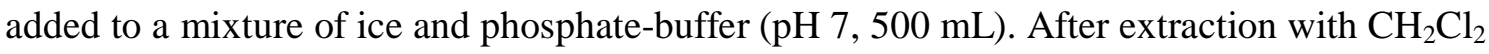
$(2 \times 500 \mathrm{~mL})$, the combined organic extracts were dried under ice-cooling over $\mathrm{Na}_{2} \mathrm{SO}_{4}$. Filtration over a short bed of Celite and removing of the solvents under vacuum at room temperature afforded $32.2 \mathrm{~g}(98 \%)$ of a colorless oil. 
$\mathbf{R}_{f}\left(\mathrm{SiO}_{2}, \mathrm{EtOAc} / n\right.$-hexane 1:6) $=0.29$

IR (ATR): $\tilde{v}\left[\mathrm{~cm}^{-1}\right]=2958$ (s), 2934 (s), 2845 (w), 2148 (w), 1673 (ss), $1580(\mathrm{w}), 1558$ (m), 1478 (s), 1425 (m), 1386 (s), 1339 (s), 1250 (s), 1197 (m), 1163 (m), 1100 (ss), 1047 (m), 1006 (s), 845 (ss), 761 (m), 703 (w).

${ }^{1} \mathbf{H}-\mathbf{N M R}\left(300 \mathrm{MHz}, \mathrm{CDCl}_{3}\right): \delta[\mathrm{ppm}]=0.21\left(\mathrm{~s}, 9 \mathrm{H}, \mathrm{SiMe}_{3}\right), 2.86(\mathrm{~m}, 2 \mathrm{H}), 3.05(\mathrm{~m}, 2 \mathrm{H})$, 3.81 (s, 6H, $2 \mathrm{OMe}$ ), 3.84 (s, 3H, OMe), 6.65 (s, 1H, 6'-H).

${ }^{13}$ C-NMR (75 MHz, $\left.\mathrm{CDCl}_{3}\right): \delta[\mathrm{ppm}]=-0.79\left(\mathrm{q}, \mathrm{SiMe}_{3}\right), 34.97(\mathrm{t}), 45.43(\mathrm{t}), 56.09(\mathrm{q}$, OMe), 60.69 (q, OMe), 60.91 (q, OMe), 87.67 (s), 98.44 (s), 101.71 (s), 109.10 (d, C-6'), 138.46 (s), 140.54 (s), 153.13 (s), 153.57 (s), 186.11 (s, C-3).

MS (DIP-EI70 eV): m/z (\%) = 446 (45) [M] $]^{+}, 374$ (17), 319 (100) [-I], 307 (34), 277 (41), 247 (9), 194 (18), 125 (47), 97 (43), 83 (40).

HRMS (EI): calc. for $[\mathrm{M}]]^{+}\left({ }^{12} \mathrm{C}_{17} \mathrm{H}_{23}{ }^{127} \mathrm{I}^{16} \mathrm{O}_{4}{ }^{28} \mathrm{Si}\right)$ : 446.0410 , found: 446.041 .

(3R)-5-(2-Iodo-3,4,5-trimethoxyphenyl)-1-(trimethylsilyl)pent-1-yn-3-ol (8)

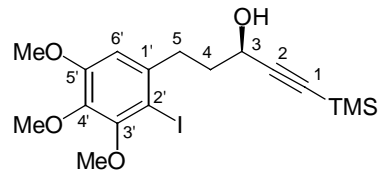

To a solution of alkynone (6) $(31.8 \mathrm{~g}, 71.2 \mathrm{mmol})$ in $i \mathrm{PrOH}(71 \mathrm{~mL}$, HPLC-grade) under argon was added $1 \mathrm{~mol} \%$ of $(R, R)$-ruthenium-catalyst (7) $(427 \mathrm{mg}, 712 \mu \mathrm{mol})$. The color of the solution turns to brown with the total dissolution of the catalyst. After $16 \mathrm{~h}$, the solvent was removed under vacuum and the residu was purified by flash-chromatography (EtOAc/cyclohexane 1:5, 1:4, 1:3). Crystallisation from $n$-hexane afforded $30.5 \mathrm{~g}$ (96\%) of colorless quadratic crystalline solid.

$\mathbf{R}_{f}\left(\mathrm{SiO}_{2}\right.$, EtOAc/cyclohexane 1:3) $=0.26$

mp.: $75^{\circ} \mathrm{C}$ ( $n$-hexane)

IR (ATR): $\tilde{v}\left[\mathrm{~cm}^{-1}\right]=3425$ (ss, br.), 2954 (m), 2935 (m), 2169 (w), 1560 (m), 1478 (s), 1425 (m), 1358 (s), 1332 (s), 1247 (s), 1197 (m), 1162 (m), 1103 (s), 1063 (m), 1042 (m), 1005 (s), 841 (ss), 759 (m).

${ }^{1} \mathbf{H}$-NMR $\left(300 \mathrm{MHz}, \mathrm{CDCl}_{3}\right): \delta[\mathrm{ppm}]=0.17\left(\mathrm{~s}, 9 \mathrm{H}, \mathrm{SiMe}_{3}\right), 1.91\left(\mathrm{~d},{ }^{3} J=5.5 \mathrm{~Hz}, 1 \mathrm{OH}\right)$, $1.95\left(\mathrm{~m}, 2 \mathrm{H}, 4-\mathrm{H}_{2}\right), 2.89$ (m, 2H, 5- $\left.\mathrm{H}_{2}\right), 3.82$ (s, 3H, OMe), 3.83 (s, 3H, OMe), 3.85 (s, 3H, $\mathrm{OMe}), 4.38\left(\mathrm{~d} \psi \mathrm{t}, J_{\mathrm{d}}=5.5 \mathrm{~Hz}, J_{\mathrm{t}}=6.5 \mathrm{~Hz}, 1 \mathrm{H}, 3-\mathrm{H}\right), 6.65\left(\mathrm{~s}, 1 \mathrm{H}, 6^{\prime}-\mathrm{H}\right)$. 
${ }^{13}$ C-NMR $\left(75 \mathrm{MHz}, \mathrm{CDCl}_{3}\right): \delta[\mathrm{ppm}]=-0.11\left(\mathrm{q}, \mathrm{SiMe}_{3}\right), 36.67(\mathrm{t}), 37.81(\mathrm{t}), 56.11(\mathrm{q}$, OMe), 60.69 (q, OMe), 60.94 (q, OMe), 62.09 (d, C-3), 87.96 (s), 90.05 (s), 106.23 (s), 108.90 (d, C-6'), 139.59 (s), 140.40 (s), 153.11 (s), 153.53 (s).

MS (DIP-EI70 eV): m/z (\%) = 448 (7) [M] ${ }^{+}, 321$ (13) [-I], 308 (20), 277 (7), 205 (15), 191 (6), 181 (100), 165 (5), 99 (5), 75 (7), 73 (16).

HRMS (EI): calc. for $[\mathrm{M}]]^{+}\left({ }^{12} \mathrm{C}_{17} \mathrm{H}_{25}{ }^{127} \mathrm{I}^{16} \mathrm{O}_{4}{ }^{28} \mathrm{Si}\right): 448.0567$, found.: 448.056 .

$[\alpha]_{\mathbf{D}}=-21.5,[\alpha]_{546}=-26.2,[\alpha]_{405}=-59.8,[\alpha]_{365}=-83.0\left(c=1.00, \mathrm{CHCl}_{3}, 20^{\circ} \mathrm{C}\right)$.

HPLC (Diacel Chiralpak AD-H, hexane/ $i \mathrm{PrOH} 98: 2, \mathrm{v}=1.0 \mathrm{ml} / \mathrm{min}, \lambda=254 \mathrm{~nm}, \sim 25^{\circ} \mathrm{C}$ ): $t_{\mathrm{R}}=20.36 \min (100 \%),>99 \% e e$.

tert-Butyl(dimethyl)silyl[(1R)-1-[(2-iodo-3,4,5-trimethoxyphenyl)ethyl]-3(trimethylsilyl)prop-2-ynyl]ether (9)

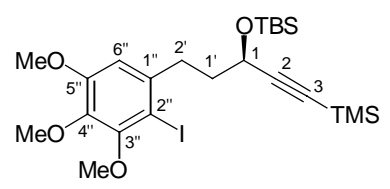

Propargylalcohol (8) (30.0 g, $66.9 \mathrm{mmol})$, imidazole (11.4 g, $167 \mathrm{mmol}, 2.5$ equiv.) and TBSCl (12.1 g, $80.3 \mathrm{mmol}, 1.2$ equiv.) were solubilized in abs. DMF (100 mL) and stirred under argon overnight. $\mathrm{H}_{2} \mathrm{O}(300 \mathrm{~mL})$ was then added and the resulting mixture was extracted with MTBE $(3 \times 300 \mathrm{~mL})$. The combined organic extracts were washed with saturated $\mathrm{NH}_{4} \mathrm{Cl}$ $(300 \mathrm{~mL})$ and dried over $\mathrm{Na}_{2} \mathrm{SO}_{4}$. After filtration over a short bed of Celite and removal of the solvent under vacuum, the raw product was purified by flash-chromatography (EtOAc/cyclohexane 1:20). Subsequent crystallisation from $n$-hexane afforded $35.1 \mathrm{~g}$ (93\%) of colorless crystals.

$\mathbf{R}_{\boldsymbol{f}}\left(\mathrm{SiO}_{2}, \mathrm{EtOAc} / n\right.$-hexane $\left.1: 10\right)=0.33$

mp.: $50-51{ }^{\circ} \mathrm{C}$ (n-hexane)

IR (ATR): $\tilde{v}\left[\mathrm{~cm}^{-1}\right]=2953$ (s), 2931 (s), $2852(\mathrm{~m}), 2169$ (w), $1561(\mathrm{w}), 1478(\mathrm{~s}), 1425(\mathrm{~m})$, 1385 (s), 1332 (m), 1248 (s), 1197 (m), 1162 (m), 1103 (ss), 1006 (s), 838 (ss), 776 (m).

${ }^{1}$ H-NMR (300 MHz, $\mathrm{CDCl}_{3}$ ): $\delta$ [ppm] = 0.13 (s, 3H, OSiMe), 0.15 (s, 3H, OSiMe), 0.15 (s, 9H, $\left.\mathrm{SiMe}_{3}\right), 0.91$ (s, 9H, OSitBu), 1.91 (m, 2H, 1'- $\left.\mathrm{H}_{2}\right), 2.84\left(\mathrm{~m}, 2 \mathrm{H}, 2\right.$ '- $\left.\mathrm{H}_{2}\right), 3.83$ (s, 6H, 2 OMe), 3.85 (s, 3H, OMe), 4.40 ( $\left.\psi \mathrm{t},{ }^{3} J=6.5 \mathrm{~Hz}, 1 \mathrm{H}, 1-\mathrm{H}\right), 6.62$ (s, 1H, 6"'-H).

${ }^{13}$ C-NMR (75 MHz, $\left.\mathrm{CDCl}_{3}\right): \delta[\mathrm{ppm}]=-4.83$ (q, OSiMe), -4.36 (q, OSiMe), -0.16 (q, $\mathrm{SiMe}_{3}$ ), 18.30 (s, OSiCMe ), 25.86 (q, OSiCMe $), 37.03$ (d), 38.77 (d), 56.11 (q, OMe), 60.68 
(q, OMe), 60.95 (q, OMe), 62.82 (d, C-1), 88.03 (s), 89.13 (s), 107.21 (s), 108.78 (d, C-6'), 140.16 (s), 140.30 (s), 153.08 (s), 153.50 (s).

MS (DIP-EI, $70 \mathrm{eV}): m / z(\%)=562(6)[\mathrm{M}]^{+}, 505$ (9) $\left[-\mathrm{C}_{4} \mathrm{H}_{9}\right], 435$ (18) [-I], 420 (14), 378 (32), 363 (100), 348 (14), 307 (78), 305 (41), 181 (61), 155 (20), 75 (14), 73 (26).

HRMS (EI): calc. for $[\mathrm{M}]]^{+}\left({ }^{12} \mathrm{C}_{23} \mathrm{H}_{39}{ }^{127} \mathrm{I}^{16} \mathrm{O}_{4}{ }^{28} \mathrm{Si}_{2}\right): 562.1432$, found.: 562.142 .

$[\alpha]_{\mathrm{D}}=+8.2,[\alpha]_{546}=+9.3,[\alpha]_{405}=+15.3,[\alpha]_{365}=+17.1\left(\mathrm{c}=0.995, \mathrm{CHCl}_{3}, 20^{\circ} \mathrm{C}\right)$.

\section{4-[6-((3R)-3-[[tert-Butyl(dimethyl)silyl]oxy]pent-4-ynyl)-2,3,4-trimethoxyphenyl]-4-oxo- butyric acid (10)}

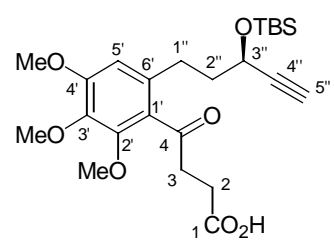

To a solution of aryl iodide $(\mathbf{9})(14.7 \mathrm{~g}, 26.1 \mathrm{mmol})$ in abs. THF $(275 \mathrm{~mL})$ under argon was added dropwise a solution of $i \operatorname{PrMgCl}\left(26.1 \mathrm{~mL}, 52.2 \mathrm{mmol}, 2\right.$ equiv., $2 \mathrm{M}$ in THF) at $-25^{\circ} \mathrm{C}$. After $4 \mathrm{~h}$, the Grignard solution was cooled to $-40{ }^{\circ} \mathrm{C}$ and added rapidly through a canula to a stirred suspension of succinic anhydride (10.4 g, 104 mmol, 4 equiv.) in abs. THF (275 mL). The resulting mixture was warmed to $\mathrm{RT}$ over $1.5 \mathrm{~h}$, added to a of saturated $\mathrm{NH}_{4} \mathrm{Cl}$ solution (450 mL) and extracted with $\mathrm{CH}_{2} \mathrm{Cl}_{2}(3 \times 250 \mathrm{~mL})$. The combined organic extracts were dried over $\mathrm{Na}_{2} \mathrm{SO}_{4}$. After filtration and removal of the solvent under vacuum, the raw product was dissolved in abs. $\mathrm{MeOH}(750 \mathrm{~mL}) . \mathrm{K}_{2} \mathrm{CO}_{3}(34.8 \mathrm{~g}, 261 \mathrm{mmol}, 10$ equiv.) was added to the resulting solution and the mixture was stirred $1.5 \mathrm{~h}$ at $\mathrm{RT}$, then added to a saturated $\mathrm{NH}_{4} \mathrm{Cl}$ solution $(750 \mathrm{~mL})$ and extracted $\mathrm{CH}_{2} \mathrm{Cl}_{2}(3 \times 300 \mathrm{~mL})$. The combined organic extracts were dried over $\mathrm{Na}_{2} \mathrm{SO}_{4}$. After filtration and removal of the solvent under vacuum, the raw product was purified by flash-chromatography (EtOAc/cyclohexane 1:3, 1:2, 1:1, 2:1) to afford $8.91 \mathrm{~g}$ (73\%) of a colorless viscose oil.

$\mathbf{R}_{f}\left(\mathrm{SiO}_{2}\right.$, EtOAc/cyclohexane 1:1) $=0.35$

IR (ATR): $\tilde{v}\left[\mathrm{~cm}^{-1}\right]=3282$ (m), 2930 (s), 2854 (w), 2109 (w), 1734 (m), 1708 (ss), 1593 (m), 1493 (m), 1455 (m), 1399 (s), 1335 (s), 1249 (s), 1129 (s), 1091 (s), 999 (m), 836 (s), 777 (m), $667(\mathrm{~m})$.

${ }^{1}$ H-NMR (500 MHz, $\left.\mathrm{CDCl}_{3}\right): \delta[\mathrm{ppm}]=0.09$ (s, 3H, OSiMe), 0.12 (s, 3H, OSiMe), 0.88 (s,

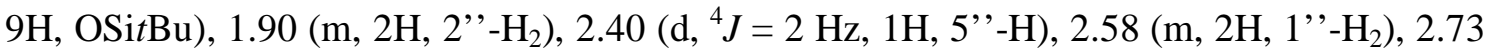
$\left(\mathrm{m}, 2 \mathrm{H}, 2-\mathrm{H}_{2}\right), 3.10\left(\mathrm{~m}, 2 \mathrm{H}, 3-\mathrm{H}_{2}\right), 3.83$ (s, 3H, OMe at C-3'), 3.837 ${ }^{* *}$ (s, 3H, OMe at C-4'), 
$\left.3.839^{* *}\right)\left(\mathrm{s}, 3 \mathrm{H}, \mathrm{OMe}\right.$ at $\left.\mathrm{C}-2{ }^{\prime}\right), 4.34\left(\mathrm{dt},{ }^{4} \mathrm{~J}=2 \mathrm{~Hz},{ }^{3} \mathrm{~J}=6.5 \mathrm{~Hz}, 1 \mathrm{H}, 3{ }^{\prime}{ }^{\prime}-\mathrm{H}\right), 6.50(\mathrm{~s}, 1 \mathrm{H}$, $\left.\left.5^{\prime}-\mathrm{H}\right){ }^{*}\right)$

${ }^{13}$ C-NMR (75 MHz, $\left.\mathrm{CDCl}_{3}\right): \delta$ [ppm] $=-5.09$ (q, OSiMe), -4.59 (q, OSiMe), 18.18 (s, $\mathrm{OSiCMe}_{3}$ ), 25.74 (q, OSiCMe $e_{3}$ ), 28.12 (t, C-2), 28.67 (t, C-1'), 39.51 (t, C-3), 40.52 (t, $\mathrm{C}-2$ '”), 56.01**) (q, OMe at C-4'), 60.88 (q, OMe at C-3'), 61.67**) (q, OMe at C-2'), 62.26 (d, C-3'’), 72.56 (d, C-5'), 85.02 (d, C-4’'), 108.73 (d, C-5'), 127.94 (s, C-1'), 134.81 (s, C-6'), 139.75 (s, C-3'), $150.62^{* *)}$ (s, C-2'), 154.31**) (s, C-4'), 178.52 (s, C-1), 203.89 (s, C-4). ${ }^{*}$

MS (DIP-EI, $70 \mathrm{eV}): m / z(\%)=407$ (16) $\left[\mathrm{M}^{+}-\mathrm{C}_{4} \mathrm{H}_{9}\right], 307$ (21), 287 (16), 263 (12), 250 (44), 235 (17), 231 (26), 219 (14), 182 (100), 167 (21), 151 (29), 101 (18), 97 (21), 83 (20).

HRMS (EI): calc. for $[\mathrm{M}]^{+}\left({ }^{12} \mathrm{C}_{20} \mathrm{H}_{27}{ }^{16} \mathrm{O}_{7}{ }^{28} \mathrm{Si}\right): 407.1526$, found.: 407.153 .

$[\alpha]_{\mathrm{D}}=+12.6,[\alpha]_{546}=+14.1,[\alpha]_{405}=+26.7,[\alpha]_{365}=+38.0\left(c=1.00, \mathrm{CHCl}_{3}, 20^{\circ} \mathrm{C}\right)$.

*) The peaks in ${ }^{1} \mathrm{H}-\mathrm{NMR}$ - and ${ }^{13} \mathrm{C}-\mathrm{NMR}$-Spectrum were assignated with the help of H,H-Cosy-, HMQC-, HMBC- und NOESY-spectra.

**) The assignments of the peaks at 3.837/56.01/154.31 ppm und 3.839/61.67/150.62 ppm in ${ }^{1} \mathrm{H}$-NMR- und ${ }^{13} \mathrm{C}$-NMR-spectra were made based on the comparison with the corresponding signals of $\mathbf{1 3 .}$

(3R)-5-Diazo-1-[6-[3-[[tert-butyl(dimethyl)silyl]oxy]pent-4-ynyl]-2,3,4-trimethoxyphenyl]-pentan-1,4-dione (11)

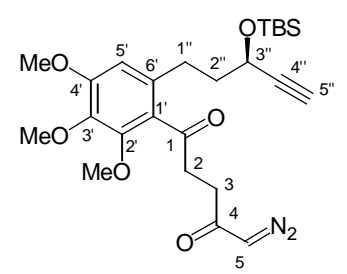

To a solution of $\gamma$-oxocarboxylic acid (10) $(8.81 \mathrm{~g}, 19.0 \mathrm{mmol})$ in THF $(160 \mathrm{~mL})$ and $\mathrm{Et}_{2} \mathrm{O}$ $(160 \mathrm{~mL})$ under argon were sequently added at $-20{ }^{\circ} \mathrm{C} \mathrm{NEt}_{3}(2.65 \mathrm{~mL}, 19.0 \mathrm{mmol}, 1.0$ equiv.) and $\mathrm{ClC}(\mathrm{O}) \mathrm{O} i \mathrm{Bu}(2.49 \mathrm{~mL}, 19.0 \mathrm{mmol}, 1.0 \mathrm{eq})$. After $1 \mathrm{~h}$, the mixture was warmed to $-10^{\circ} \mathrm{C}$ and a solution of $\mathrm{CH}_{2} \mathrm{~N}_{2}\left(380 \mathrm{~mL}, 87.4 \mathrm{mmol}, 4.6 \mathrm{eq}, 0.23 \mathrm{M}\right.$ in $\left.\mathrm{Et}_{2} \mathrm{O}\right)$ was added dropwise. After stirring at $-5{ }^{\circ} \mathrm{C}$ overnight, the solution was warmed to RT and silica was added to destroy the excess of diazomethane. After filtration, the solution was washed with water (200 $\mathrm{mL})$, saturated $\mathrm{NaHCO}_{3}(200 \mathrm{~mL})$ and brine $(200 \mathrm{~mL})$. The organic phase was dried over $\mathrm{Na}_{2} \mathrm{SO}_{4}$ and the solvent was removed under vacuum. Purification by flash-chromatography (EtOAc/cyclohexane 1:5, 1:4, 1:3, 1:2) afforded $6.59 \mathrm{~g}(71 \%)$ of a light yellow oil. 
$\mathbf{R}_{f}\left(\mathrm{SiO}_{2}\right.$, EtOAc/cyclohexane 1:3) $=0.24$

IR (ATR): $\tilde{v}\left[\mathrm{~cm}^{-1}\right]=3272(\mathrm{w}), 3095(\mathrm{w}), 2928(\mathrm{~s}), 2853(\mathrm{~m}), 2100(\mathrm{ss}, \mathrm{C}=\mathrm{N}=\mathrm{N}), 1696(\mathrm{~m})$, 1643 (s), 1593 (m), 1571 (w), 1492 (m), 1461 (s), 1400 (s), 1376 (s), 1344 (ss), 1316 (s), 1250 (s), 1128 (s), 1093 (ss), 835 (ss), 777 (s), 665 (w).

${ }^{1} \mathbf{H}-\mathbf{N M R}\left(500 \mathrm{MHz}, \mathrm{CDCl}_{3}\right.$ ): $\delta$ [ppm] $=0.09$ (s, 3H, OSiMe), 0.12 (s, 3H, OSiMe), 0.88 (s, 9H, OSitBu), 1.89 (m, 2H, 2' '- $\mathrm{H}_{2}$ ), 2.38 (d, ${ }^{4} \mathrm{~J}=2 \mathrm{~Hz}, 1 \mathrm{H}, 5$ '’-H), 2.58 (m, 2H, 1' $\left.-\mathrm{H}_{2}\right), 2.68$ (br. m, 2H, 3- $\mathrm{H}_{2}$ ), 3.10 (m, 2H, 2- $\mathrm{H}_{2}$ ), 3.80 (s, 3H, OMe at C-3'), 3.82 (s, 3H, OMe at C-4'), 3.83 (s, 3H, OMe at C-2'), 4.36 (dt, $\left.{ }^{4} J=2 \mathrm{~Hz},{ }^{3} J=6.5 \mathrm{~Hz}, 1 \mathrm{H}, 3{ }^{\prime}{ }^{-}-\mathrm{H}\right), 5.31$ (br. s, $\left.1 \mathrm{H}, 5-\mathrm{H}\right)$, $\left.6.48\left(\mathrm{~s}, 1 \mathrm{H}, 5^{\prime}-\mathrm{H}\right){ }^{*}\right)$

${ }^{13}$ C-NMR (62.5 MHz, $\mathrm{CDCl}_{3}$ ): $\delta$ [ppm] = -5.10 (q, OSiMe), -4.64 (q, OSiMe), 18.15 (s, $\mathrm{OSiCMe}_{3}$ ), 25.72 (q, OSiCMe $), 28.70$ (t, C-1'”), 34.23 (br. t, C-3), 39.54 (br. t, C-2), 40.52 (t, C-2'’), 54.39 (br. d, C-5), 55.96 (q, OMe at C-4'), 60.81 (q, OMe at C-3'), 61.59 (q, OMe at C-2'), 62.26 (d, C-3'”), 72.45 (d, C-5'), 85.05 (d, C-4'’), 108.64 (d, C-5'), 128.04 (s, C-1'), 134.66 (s, C-6'), 139.65 (s, C-3'), 150.49 (s, C-2'), 154.21 (s, C-4'), 193.15 (s, C-4), 204.41 $\left.(\mathrm{C}-1){ }^{*}\right)$

MS (DIP-EI, $70 \mathrm{eV}): m / z(\%)=488(4)[\mathrm{M}]^{+}, 460(45)\left[-\mathrm{N}_{2}\right], 403(10)\left[-\mathrm{N}_{2}-\mathrm{C}_{4} \mathrm{H}_{9}\right], 351$ (9), 305 (7), 231 (56), 219 (15), 207 (14), 181 (20), 129 (48), 115 (15), 83 (30), 75 (92), 73 (86).

HRMS (EI): calc. for $[\mathrm{M}]^{+}\left({ }^{12} \mathrm{C}_{25} \mathrm{H}_{36}{ }^{14} \mathrm{~N}_{2}{ }^{16} \mathrm{O}_{6}{ }^{28} \mathrm{Si}\right): 488.2343$, found.: 488.235 .

$[\alpha]_{\mathrm{D}}=+11.3,[\alpha]_{546}=+13.0\left(c=1.00, \mathrm{CHCl}_{3}, 20^{\circ} \mathrm{C}\right)$.

*) Peaks in ${ }^{1} \mathrm{H}$-NMR- und ${ }^{13} \mathrm{C}$-NMR-spectra were assigned with the help of H,H-Cosy-, HMQC-, HMBC- und NOESY-spectra.

$(7 R, 9 R, 12 \mathrm{a} R)-7-[[$ tert-Butyl(dimethyl)silyl]oxy]-1,2,3-trimethoxy-9,12a-epoxy$6,7,9,11,12,12 a-h e x a h y d r o b e n z o[a] h e p t a l e n-10(5 H)$-one $(12)$

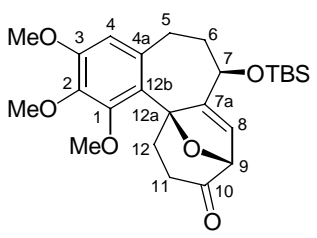

To a stirred suspension of $\mathrm{Rh}_{2}(\mathrm{OAc})_{4}(132 \mathrm{mg}, 298 \mu \mathrm{mol}, 3 \mathrm{~mol} \%)$ in abs. toluene $(200 \mathrm{~mL})$, was added over $6 \mathrm{~h}$ through via a syringe pump a solution of diazoketone (11) (4.86 g, $9.95 \mathrm{mmol})$ in abs. toluene $(300 \mathrm{~mL})$. After $60 \mathrm{~min}$ under reflux, the solution was cooled to RT and the solvent was removed under vacuum. The raw product was purified by flashchromatography (EtOAc/cyclohexane 1:5) to afford $2.94 \mathrm{~g}(64 \%)$ of a colorless viscose oil.

$\mathbf{R}_{f}\left(\mathrm{SiO}_{2}\right.$, EtOAc/cyclohexane 1:5) $=0.24$ 
IR (ATR): $\tilde{v}\left[\mathrm{~cm}^{-1}\right]=2932(\mathrm{~m}), 2854(\mathrm{w}), 1726$ (ss, C=O ketone), $1592(\mathrm{~m}), 1493(\mathrm{~m}), 1451$ (s), 1402 (m), 1347 (m), 1320 (m), 1291 (m), 1249 (s), 1197 (w), 1129 (s), 1087 (ss), 1031 (m), 1003 (m), 921 (m), 881 (m), 834 (s), 807 (m), 775 (m), $666(\mathrm{w})$.

${ }^{1} \mathbf{H}-\mathbf{N M R}\left(500 \mathrm{MHz}, \mathrm{CDCl}_{3}\right.$ ): $\delta$ [ppm] = 0.01 (s, 3H, OSiMe), 0.03 (s, 3H, OSiMe), 0.88 (s, 9H, OSitBu), $1.83\left(\mathrm{ddd},{ }^{3} J_{1}=6.5 \mathrm{~Hz},{ }^{3} J_{2}=7.5 \mathrm{~Hz},{ }^{2} J=13.5 \mathrm{~Hz}, 1 \mathrm{H}, 12-\mathrm{H}_{\mathrm{a}}\right.$ ), 1.86 (dddd, $\left.{ }^{3} J_{1}=4.0 \mathrm{~Hz},{ }^{3} J_{2}=4.5 \mathrm{~Hz},{ }^{3} J_{3}=5.5 \mathrm{~Hz},{ }^{2} J=13.5 \mathrm{~Hz}, 1 \mathrm{H}, 6-\mathrm{H}_{\mathrm{a}}\right), 2.32$ (dddd, ${ }^{3} J_{1}=3.5 \mathrm{~Hz}$,

$\left.{ }^{3} J_{2}=8.5 \mathrm{~Hz},{ }^{3} J_{3}=13.5 \mathrm{~Hz},{ }^{2} J=13.5 \mathrm{~Hz}, 1 \mathrm{H}, 6-\mathrm{H}_{\mathrm{b}}\right), 2.41\left(\mathrm{ddd},{ }^{3} J_{1}=3.5 \mathrm{~Hz},{ }^{3} J_{2}=4 \mathrm{~Hz}\right.$, $\left.{ }^{2} J=14.5 \mathrm{~Hz}, 1 \mathrm{H}, 5-\mathrm{H}_{\mathrm{a}}\right), 2.60\left(\mathrm{ddd},{ }^{3} J_{1}=3.5 \mathrm{~Hz},{ }^{3} J_{2}=7.5 \mathrm{~Hz},{ }^{2} J=17 \mathrm{~Hz}, 1 \mathrm{H}, 11-\mathrm{H}_{\mathrm{a}}\right), 2.92$ $\left(\mathrm{ddd},{ }^{3} J_{1}=6.5 \mathrm{~Hz},{ }^{3} J_{2}=9 \mathrm{~Hz},{ }^{2} J=17 \mathrm{~Hz}, 1 \mathrm{H}, 11-\mathrm{H}_{\mathrm{b}}\right), 3.08\left(\mathrm{ddd},{ }^{3} J_{1}=4.5 \mathrm{~Hz},{ }^{3} J_{2}=13.5 \mathrm{~Hz}\right.$, $\left.{ }^{2} J=14.5 \mathrm{~Hz}, 1 \mathrm{H}, 5-\mathrm{H}_{\mathrm{b}}\right), 3.33\left(\mathrm{ddd},{ }^{3} J_{1}=3.5 \mathrm{~Hz},{ }^{3} J_{2}=9 \mathrm{~Hz},{ }^{2} J=13.5 \mathrm{~Hz}, 1 \mathrm{H}, 12-\mathrm{H}_{\mathrm{b}}\right), 3.83$ (s, $3 \mathrm{H}, \mathrm{OMe}$ at C-3), 3.85 (s, 3H, OMe at C-2), 3.95 (s, 3H, OMe at C-1), $4.51\left(\mathrm{ddd},{ }^{4} \mathrm{~J}=2 \mathrm{~Hz}\right.$, $\left.{ }^{3} J_{1}=5.5 \mathrm{~Hz},{ }^{3} J_{2}=8.5 \mathrm{~Hz}, 1 \mathrm{H}, 7-\mathrm{H}\right), 4.72\left(\mathrm{~d},{ }^{3} J=2 \mathrm{~Hz}, 1 \mathrm{H}, 9-\mathrm{H}\right), 6.00(\psi \mathrm{t}, J=2 \mathrm{~Hz}, 1 \mathrm{H}$, C-8), 6.39 (s, 1H, 4-H). ${ }^{*}$

${ }^{13}$ C-NMR (75 MHz, $\left.\mathrm{CDCl}_{3}\right): \delta[\mathrm{ppm}]=-4.83\left(\mathrm{q}, \mathrm{OSiMe}_{2}\right), 18.05\left(\mathrm{~s}, \mathrm{OSiCMe}_{3}\right), 25.72(\mathrm{q}$, $\mathrm{OSiCMe}_{3}$ ), 30.55 (t, C-12), 32.20 (t, C-5), 35.63 (t, C-11), 37.00 (t, C-6), 55.84 (q, OMe an C-3), 60.74 (q, OMe an C-2), 62.29 (q, OMe an C-1), 67.46 (d, C-7), 85.55 (d, C-9), 88.30 (s, C-12a), 109.68 (d, C-4), 120.05 (d, C-8), 123.69 (s, C-12b), 137.12 (s, C-4a), 141.86 (s, C-2), 153.27 (s, C-3), 154.88 (s, C-1), 156.54 (s, C-7a), 204.41 (s, C-10). ${ }^{*}$

MS (DIP-EI, $70 \mathrm{eV}): m / z(\%)=460(15)[\mathrm{M}]^{+}, 403$ (100) $\left[-\mathrm{C}_{4} \mathrm{H}_{9}\right], 375$ (16), 361 (9), 329 (40), 285 (9), 273 (58), 181 (11), 75 (16), 73 (18).

HRMS (EI): calc. for $[\mathrm{M}]{ }^{+}\left({ }^{12} \mathrm{C}_{25} \mathrm{H}_{36}{ }^{16} \mathrm{O}_{6}{ }^{28} \mathrm{Si}\right): 460.2281$, found.: 460.228 .

$[\alpha]_{\mathrm{D}}=+125.5, \quad[\alpha]_{546}=+161.9, \quad[\alpha]_{405}=+660.5, \quad[\alpha]_{365}=+1450[\alpha]_{334}=+5110 \quad(c=1.05$, $\left.\mathrm{CHCl}_{3}, 20^{\circ} \mathrm{C}\right)$.

*) Peaks in ${ }^{1} \mathrm{H}-\mathrm{NMR}$ - und ${ }^{13} \mathrm{C}$-NMR-spectra were assigned with the help of $\mathrm{H}, \mathrm{H}-\mathrm{Cosy}-$, HMQC-, HMBC- und NOESY-spectra.

$(7 R, 9 R, 12 \mathrm{a} R)$-7-Hydroxy-1,2,3-trimethoxy-9,12a-epoxy-6,7,9,11,12,12ahexahydrobenzo[ $[a]$ heptalen-10 $(5 H)$-one $(13)$

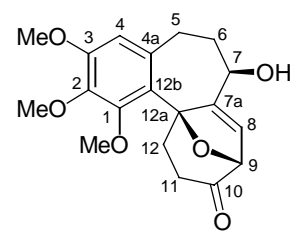

To a solution of silylether (12) $(98 \mathrm{mg}, 213 \mu \mathrm{mol})$ in abs. THF ( $2 \mathrm{~mL})$ under argon was added at $0{ }^{\circ} \mathrm{C}$ a solution of TBAF $(0.64 \mathrm{~mL}, 640 \mu \mathrm{mol}, 3$ equiv., $1 \mathrm{M}$ in THF). After $30 \mathrm{~min}$, water 
(10 mL) was added and the solution was extracted with $\mathrm{CH}_{2} \mathrm{Cl}_{2}(3 \times 4 \mathrm{~mL})$. The combined organic extracts were filtered over a short bed of Celite in a Pasteur-pipette and the solvent was removed under vacuum. After radial chromatography over silica-gel (EtOAc/cyclohexane 2:1), $68 \mathrm{mg}(92 \%)$ of a colorless solid were obtained.

$\mathbf{R}_{f}\left(\mathrm{SiO}_{2}\right.$, EtOAc/cyclohexane 3:1) $=0.36$

mp.: $154-155^{\circ} \mathrm{C}$ (EtOAc/cyclohexane)

IR (ATR): $\tilde{v}\left[\mathrm{~cm}^{-1}\right]=3443$ (s, br., OH), 2936 (m), 2854 (w), 1722 (ss, C=O ketone), 1592 (m), 1492 (m), 1451 (s), 1401 (m), 1320 (m), 1241 (w), 1195 (w), 1127 (s), 1088 (m), 1027 (s), $1002(\mathrm{~m}), 918(\mathrm{w}), 833(\mathrm{w}), 808(\mathrm{w}), 750(\mathrm{~s}), 665(\mathrm{w})$.

${ }^{1}$ H-NMR (500 MHz, $\mathrm{CDCl}_{3}$ ): $\delta$ [ppm] = $1.84\left(\mathrm{~m}, 1 \mathrm{H}, 12-\mathrm{H}_{\mathrm{a}}\right), 1.87\left(\mathrm{~m}, 1 \mathrm{H}, 6-\mathrm{H}_{\mathrm{a}}\right), 2.37$ (dddd, $\left.{ }^{3} J_{1}=3.5 \mathrm{~Hz},{ }^{3} J_{2}=8 \mathrm{~Hz},{ }^{3} J_{3}=13 \mathrm{~Hz},{ }^{2} J=13.5 \mathrm{~Hz}, 1 \mathrm{H}, 6-\mathrm{H}_{\mathrm{b}}\right), 2.46$ (ddd, ${ }^{3} J_{1}=3.5 \mathrm{~Hz}$, $\left.{ }^{3} J_{2}=4 \mathrm{~Hz},{ }^{2} J=14.5 \mathrm{~Hz}, 1 \mathrm{H}, 5-\mathrm{H}_{\mathrm{a}}\right), 2.60\left(\mathrm{ddd},{ }^{3} J_{1}=3 \mathrm{~Hz},{ }^{3} J_{2}=7.5 \mathrm{~Hz},{ }^{2} J=17 \mathrm{~Hz}, 1 \mathrm{H}\right.$, $\left.11-\mathrm{H}_{\mathrm{a}}\right), 2.95\left(\mathrm{ddd},{ }^{3} J_{1}=7 \mathrm{~Hz},{ }^{3} J_{2}=9.5 \mathrm{~Hz},{ }^{2} J=17 \mathrm{~Hz}, 1 \mathrm{H}, 11-\mathrm{H}_{\mathrm{b}}\right), 3.04\left(\mathrm{ddd},{ }^{3} J_{1}=4 \mathrm{~Hz}\right.$, $\left.{ }^{3} J_{2}=13 \mathrm{~Hz},{ }^{2} J=14.5 \mathrm{~Hz}, 1 \mathrm{H}, 5-\mathrm{H}_{\mathrm{b}}\right), 3.26\left(\mathrm{ddd},{ }^{3} J_{1}=3 \mathrm{~Hz},{ }^{3} J_{2}=9.5 \mathrm{~Hz},{ }^{2} J=13 \mathrm{~Hz}, 1 \mathrm{H}\right.$, 12- $\mathrm{H}_{\mathrm{b}}$ ), 3.83 (s, 3H, OMe an C-3), 3.84 (s, 3H, OMe an C-2), 3.95 (s, 3H, OMe an C-1), 4.61 (ddd, $\left.{ }^{4} J=2 \mathrm{~Hz},{ }^{3} J_{1}=5.5 \mathrm{~Hz},{ }^{3} J_{2}=8 \mathrm{~Hz}, 1 \mathrm{H}, 7-\mathrm{H}\right), 4.74\left(\mathrm{~d},{ }^{3} J=2 \mathrm{~Hz}, 1 \mathrm{H}, 9-\mathrm{H}\right), 6.06(\psi \mathrm{t}$, $\left.J=2 \mathrm{~Hz}, 1 \mathrm{H}, \mathrm{C}-8), 6.40(\mathrm{~s}, 1 \mathrm{H}, 4-\mathrm{H}){ }^{*}\right)$

${ }^{13}$ C-NMR $\left(75 \mathrm{MHz}, \mathrm{CDCl}_{3}\right): \delta$ [ppm] = $30.94(\mathrm{t}, \mathrm{C}-12), 31.87(\mathrm{t}, \mathrm{C}-5), 35.65(\mathrm{t}, \mathrm{C}-6), 36.00$ (t, C-11), 55.85 (q, OMe at C-3), 60.73 (q, OMe at C-2), 62.26 (q, OMe at C-1), 67.16 (d, C-7), 85.45 (d, C-9), 88.40 (s, C-12a), 109.69 (d, C-4), 120.10 (d, C-8), 123.82 (s, C-12b), 136.74 (s, C-4a), 141.88 (s, C-2), 153.21 (s, C-3), 154.82 (s, C-1), 156.62 (s, C-7a), 204.72 (s, C-10). ${ }^{*}$

MS (DIP-EI, $70 \mathrm{eV}): \mathrm{m} / z(\%)=346$ (38) [M] ${ }^{+}, 329$ (15), 318 (39) [-CO], 299 (20), 290 (30), 273 (21), 259 (26), 220 (33), 181 (100), 128 (23), 115 (39), 91 (20), 77 (24).

HRMS (EI): calc. for $[\mathrm{M}]{ }^{+}\left({ }^{12} \mathrm{C}_{19} \mathrm{H}_{22}{ }^{16} \mathrm{O}_{6}\right): 346.1416$, found.: 346.142 .

$[\alpha]_{\mathrm{D}}=+217.5,[\alpha]_{546}=+283.5,[\alpha]_{405}=+1117.7,[\alpha]_{365}=+2438.0\left(c=0.990, \mathrm{CHCl}_{3}, 20^{\circ} \mathrm{C}\right)$. HPLC (Macherey-Nagel Nucleosil pre-column, Diacel Chiralpak AD-H, Hexane/iPrOH $\left.90: 10, \mathrm{v}=1.0 \mathrm{ml} / \mathrm{min}, \lambda=254 \mathrm{~nm}, \sim 25^{\circ} \mathrm{C}\right): t_{\mathrm{R}}=9.45 \mathrm{~min}(100 \%),>99 \%$ ee.

*) Peaks in ${ }^{1} \mathrm{H}$-NMR- und ${ }^{13} \mathrm{C}$-NMR-spectra were assigned according to the data of the corresponding ( $\mathrm{rac}$ ) compound, with the help of H,H-Cosy-, HMQC-, HMBC- und NOESYspectra.

(7RS)-7-[[tert-Butyl(dimethyl)silyl]oxy]-1,2,3-trimethoxy-6,7-dihydrobenzo[a]heptalen10(5H)-one (rac-14) 


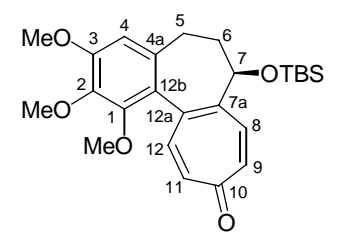

To a solution of ketone ( $\mathrm{rac}-\mathbf{1 2})(400 \mathrm{mg}, 869 \mu \mathrm{mol})$ in abs. $\mathrm{CH}_{2} \mathrm{Cl}_{2}(20 \mathrm{~mL})$ under argon was added dropwise at $-78^{\circ} \mathrm{C}$ a solution of $\mathrm{Et}_{2} \mathrm{AlCl}(4.35 \mathrm{~mL}, 4.35 \mathrm{mmol}, 5$ equiv., $1 \mathrm{M}$ in $n$ hexane). The solution was warmed to $0^{\circ} \mathrm{C}$ over $2 \mathrm{~h}$ and cooled again to $-78{ }^{\circ} \mathrm{C}$. After addition of $\mathrm{MeOH}(5 \mathrm{~mL})$, the solution was slowly warmed to $\mathrm{RT}$ and saturated $\mathrm{NH}_{4} \mathrm{Cl}(50 \mathrm{~mL})$ was added. The aqueous phase was extracted $\mathrm{CH}_{2} \mathrm{Cl}_{2}(2 \times 50 \mathrm{~mL})$ and the combined organic extracts were dried over $\mathrm{Na}_{2} \mathrm{SO}_{4}$. After filtration and removal of the solvent under vacuum, the raw product was purified by flash-chromatography (EtOAc/cyclohexane 1:4, 1:3, 1:2, 1:1) to afford $199 \mathrm{mg}(52 \%)$ of a yellow oil.

$\mathbf{R}_{f}\left(\mathrm{SiO}_{2}\right.$, EtOAc/cyclohexane 1:2) $=0.20$

IR (ATR): $\tilde{v}\left[\mathrm{~cm}^{-1}\right]=2928(\mathrm{~s}), 2853(\mathrm{~m}), 1623$ (s), 1562 (ss), $1488(\mathrm{~m}), 1454$ (s), 1401 (m), 1341 (m), 1320 (m), 1234 (m), 1134 (s), 1093 (ss), 1003 (s), 876 (s), 835 (ss), 777 (s), 730 (w), $670(\mathrm{w})$.

${ }^{1}$ H-NMR (300 MHz, $\mathrm{CDCl}_{3}$ ): $\delta$ [ppm] $=-0.13$ (s, 3H, OSiMe), -0.09 (s, 3H, OSiMe), 0.82 (s, 9H, OSitBu), $1.95\left(\mathrm{~m}, 1 \mathrm{H}, 6-\mathrm{H}_{\mathrm{a}}\right), 2.32\left(\mathrm{~m}, 1 \mathrm{H}, 6-\mathrm{H}_{\mathrm{b}}\right), 2.35\left(\mathrm{~m}, 2 \mathrm{H}, 5-\mathrm{H}_{2}\right), 3.64(\mathrm{~s}, 3 \mathrm{H}$, OMe an C-1), 3.89 (s, 3H, OMe an C-2), 3.90 (s, 3H, OMe an C-3), 4.27 (m, 1H, 7-H), 6.54 (s, 1H, 4-H), $6.95\left(\mathrm{dd},{ }^{4} \mathrm{~J}=3 \mathrm{~Hz},{ }^{3} J=12.5 \mathrm{~Hz}, 11-\mathrm{H}\right), 7.10\left(\mathrm{dd},{ }^{4} \mathrm{~J}=3 \mathrm{~Hz},{ }^{3} \mathrm{~J}=13 \mathrm{~Hz}, 9-\mathrm{H}\right)$, $\left.7.23\left(\mathrm{~d},{ }^{3} J=12.5 \mathrm{~Hz}, 12-\mathrm{H}\right), 7.77\left(\mathrm{~d},{ }^{3} J=13 \mathrm{~Hz}, 8-\mathrm{H}\right) .{ }^{*}\right)$

${ }^{13}$ C-NMR (75 MHz, $\mathrm{CDCl}_{3}$ ): $\delta[\mathrm{ppm}]=-5.09$ (q, OSiMe), -4.95 (q, OSiMe), 18.16 (s, $\mathrm{OSiCMe}_{3}$ ), 25.75 (q, OSiCMe $), 29.99$ (t, C-5), 42.16 (t, C-6), 56.01 (q, OMe an C-3), 61.00 (q, OMe an C-1), 61.23 (q, OMe an C-2), 71.80 (d, C-7), 107.24 (d, C-4), 124.93 (s, C-12b), 134.19 (d, C-8), 136.02 (s, C-4a), 138.23 (d, C-11), 139.05 (s, C-12a), 140.13 (d, C-9), 141.17 (s, C-2), 141.28 (d, C-12), 147.67 (s, C-7a), 150.72 (s, C-1), 153.96 (s, C-3), 187.43 (s, C-10). ${ }^{*}$

MS (DIP-EI, $70 \mathrm{eV}): m / z(\%)=442(23)[\mathrm{M}]^{+}, 385(100)\left[-\mathrm{C}_{4} \mathrm{H}_{9}\right], 357(59)\left[-\mathrm{C}_{4} \mathrm{H}_{9}-\mathrm{CO}\right]$, 342 (27), 252 (16), 126 (65), 73 (42).

HRMS (EI): calc. für $[\mathrm{M}]{ }^{+}\left({ }^{12} \mathrm{C}_{25} \mathrm{H}_{34}{ }^{16} \mathrm{O}_{5}{ }^{28} \mathrm{Si}\right): 442.2176$, found.: 442.217 .

*) Peaks in ${ }^{1} \mathrm{H}$-NMR- und ${ }^{13} \mathrm{C}$-NMR-spectra were assigned with the help of H,H-Cosy-, HMQC-, HMBC- und NOESY-spectra. 
$(7 R, 9 R, 10 S, 12 \mathrm{a} R)-7-[[$ tert-Butyl(dimethyl)silyl]oxy]-1,2,3-trimethoxy-9,12a-epoxy$5,6,7,9,10,11,12,12$ a-octahydrobenzo $[a]$ heptalen-10-ol (15)

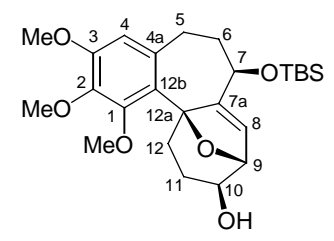

To a solution of ketone (12) $(1.40 \mathrm{~g}, 3.04 \mathrm{mmol})$ in abs. THF (30 mL) under argon was added dropwise at $-78^{\circ} \mathrm{C}$ a solution of L-Selectride (7.6 mL, 2.5 equiv., $1 \mathrm{M}$ in THF). After $3 \mathrm{~h}$, $\mathrm{MeOH}(5 \mathrm{~mL})$ was added and the reaction mixture was slowly warmed to RT, gas evolution was observed. After addition of saturated $\mathrm{NH}_{4} \mathrm{Cl}(50 \mathrm{~mL})$, the mixture was extracted with MTBE $(3 \times 50 \mathrm{~mL})$ and the combined organic extracts were dried over $\mathrm{Na}_{2} \mathrm{SO}_{4}$. After filtration and removal of the solvent under vacuum, the raw product was purified by flashchromatography (EtOAc/cyclohexane 1:4, 1:3, 1:2) to afford $1.13 \mathrm{~g}(80 \%)$ of a colorless faum.

$\mathbf{R}_{f}\left(\mathrm{SiO}_{2}, \mathrm{EtOAc} / n\right.$-hexane $\left.1: 2\right)=0.19$

IR (ATR): $\tilde{v}\left[\mathrm{~cm}^{-1}\right]=3425$ (w, br.), 2930 (ss), 2854 (m), 1591 (m), 1490 (m), 1452 (s), 1399 (s), 1346 (m), 1319 (s), 1255 (s), 1197 (m), 1130 (s), 1088 (ss), 1017 (m), 950 (w), 883 (w), 835 (ss), $774(\mathrm{~m}), 665(\mathrm{w})$.

${ }^{1}$ H-NMR (300 MHz, $\mathrm{CDCl}_{3}$ ): $\delta$ [ppm] $=0.044$ (s, 3H, OSiMe), 0.045 (s, 3H, OSiMe), 0.91 (s, 9H, OSitBu), $1.18\left(\mathrm{dd},{ }^{3} J=6.5 \mathrm{~Hz},{ }^{2} J=14 \mathrm{~Hz}, 1 \mathrm{H}, 12-\mathrm{H}_{\mathrm{a}}\right), 1.71\left(\mathrm{dd},{ }^{3} J=6.5 \mathrm{~Hz}\right.$, $\left.{ }^{2} J=14 \mathrm{~Hz}, 1 \mathrm{H}, 11-\mathrm{H}_{\mathrm{a}}\right), 1.85\left(\mathrm{~m}, 1 \mathrm{H}, 6-\mathrm{H}_{\mathrm{a}}\right), 1.88\left(\mathrm{~m}, 1 \mathrm{H}, 11-\mathrm{H}_{\mathrm{b}}\right), 2.19$ (dddd, ${ }^{3} J_{1}=3 \mathrm{~Hz}$, $\left.{ }^{3} J_{2}=7.5 \mathrm{~Hz},{ }^{3} J_{3} \mathrm{~Hz}=12.5 \mathrm{~Hz},{ }^{2} J=13.5 \mathrm{~Hz}, 1 \mathrm{H}, 6-\mathrm{H}_{\mathrm{b}}\right), 2.40\left(\mathrm{ddd},{ }^{3} J_{1}=3 \mathrm{~Hz},{ }^{3} J_{2}=5 \mathrm{~Hz}\right.$, ${ }^{2} J=14.5 \mathrm{~Hz}, 1 \mathrm{H}, 5-\mathrm{H}_{\mathrm{a}}$ ), 2.90 (br. d, $\left.J=10 \mathrm{~Hz}, 1 \mathrm{OH}\right), 3.14$ (ddd, ${ }^{3} J_{1}=4 \mathrm{~Hz},{ }^{3} J_{2}=12.5 \mathrm{~Hz}$, $\left.{ }^{2} J=14.5 \mathrm{~Hz}, 1 \mathrm{H}, 5-\mathrm{H}_{\mathrm{b}}\right), 3.35\left(\mathrm{ddd},{ }^{3} J_{1}=6.5 \mathrm{~Hz},{ }^{3} J_{2}=11.5 \mathrm{~Hz},{ }^{2} J=14 \mathrm{~Hz}, 1 \mathrm{H}, 12-\mathrm{H}_{\mathrm{b}}\right), 3.48$ (s, 1H, 10-H), 3.81 (s, 3H, OMe an C-3), 3.84 (s, 3H, OMe an C-2), 3.93 (s, 3H, OMe an C-1), $4.30\left(\mathrm{ddd},{ }^{4} J=2 \mathrm{~Hz},{ }^{3} J_{1}=5.5 \mathrm{~Hz},{ }^{3} J_{2}=7.5 \mathrm{~Hz}, 1 \mathrm{H}, 7-\mathrm{H}\right), 4.64$ (s, 1H, 9-H), 6.10 ( $\psi \mathrm{t}$, $J=2 \mathrm{~Hz}, 1 \mathrm{H}, 8-\mathrm{H}), 6.36(\mathrm{~s}, 1 \mathrm{H}, 4-\mathrm{H}){ }^{*}{ }^{*}$

${ }^{13}$ C-NMR (75 MHz, $\mathrm{CDCl}_{3}$ ): $\delta$ [ppm] = -4.88 (q, OSiMe), -4.62 (q, OSiMe), 18.06 (s, $\mathrm{OSiCMe}_{3}$ ), 22.32 (t, C-12), 25.78 (q, OSiCMe 3 ), 26.43 (t, C-11), 32.65 (t, C-5), 37.14 (t, C-6), 55.79 (q, OMe an C-3), 60.69 (q, OMe an C-2), 61.63 (q, OMe an C-1), 63.67 (d, C-10), 68.38 (d, C-7), 82.51 (d, C-9), 89.55 (s, C-12a), 109.76 (d, C-4), 122.85 (d, C-8), 124.31 (s, C-12b), 137.07 (s, C-4a), 141.91 (s, C-2), 149.12 (s, C-7a), 152.71 (s, C-3), 155.36 (s, C-1). ${ }^{*}$

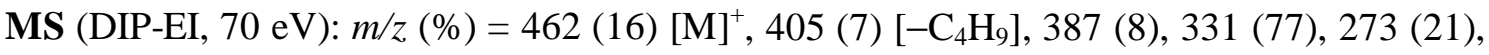
207 (26), 181 (23), 126 (20), 75 (26), 73 (38). 
$[\alpha]_{\mathrm{D}}=-103.3,[\alpha]_{546}=-123.4,[\alpha]_{405}=-262.8,[\alpha]_{365}=-355.0\left(c=1.01, \mathrm{CHCl}_{3}, 20^{\circ} \mathrm{C}\right)$.

HRMS (EI): calc. for $[\mathrm{M}]^{+}\left({ }^{12} \mathrm{C}_{25} \mathrm{H}_{38}{ }^{16} \mathrm{O}_{6}{ }^{28} \mathrm{Si}\right)$ : 462.2438, found.: 462.243.

*) Peaks in ${ }^{1} \mathrm{H}-\mathrm{NMR}$ - und ${ }^{13} \mathrm{C}$-NMR-spectra were assignated according to the data of the corresponding (rac) compound, with the help of H,H-Cosy-, HMQC-, HMBC- und NOESYspectra.

(7R,9R,10S)-7-[[tert-Butyl(dimethyl)silyl]oxy]-1,2,3-trimethoxy-5,6,7,9,10,11hexahydrobenzo[a]heptalen-9,10-diol (16)

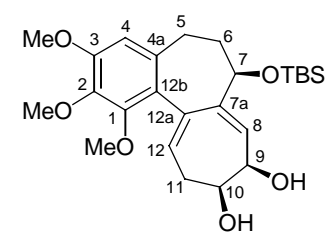

To a solution of endoxide (15) $(455 \mathrm{mg}, 983 \mu \mathrm{mol})$ in $\mathrm{CH}_{2} \mathrm{Cl}_{2}(20 \mathrm{~mL})$ under argon were subsequently dropwise added at $-50{ }^{\circ} \mathrm{C} \mathrm{NEt}_{3}(0.68 \mathrm{~mL}, 4.9 \mathrm{mmol}, 5$ equiv.) and TMSOTf (0.89 $\mathrm{mL}, 4.9 \mathrm{mmol}, 5$ equiv.). The reaction mixture was warmed to $-10^{\circ} \mathrm{C}$ over $45 \mathrm{~min}$ and then cooled to $-50^{\circ} \mathrm{C}$. Abs. $\mathrm{MeOH}(1 \mathrm{~mL})$ was then added and the reaction mixture was warmed to RT. The solvent was removed under vacuum at RT and the residue was solved in abs. $\mathrm{MeOH}(30 \mathrm{~mL}) . \mathrm{K}_{2} \mathrm{CO}_{3}\left(1.36 \mathrm{~g}, 9.84 \mathrm{mmol}, 10\right.$ equiv.) was added at $0^{\circ} \mathrm{C}$ and the reaction mixture was warmed to RT over 45 min before addition of saturated $\mathrm{NH}_{4} \mathrm{Cl}(50 \mathrm{~mL})$. After extraction with $\mathrm{CH}_{2} \mathrm{Cl}_{2}(3 \times 25 \mathrm{~mL})$, the combined organic extracts were dried over $\mathrm{Na}_{2} \mathrm{SO}_{4}$, filtered and the solvent was removed under vacuum at RT. The raw product was purified by flash-chromatography (EtOAc/cyclohexane 1:8, 1:6, 1:2, 1:1) to afford $287 \mathrm{mg}(63 \%)$ of colorless oil.

$\mathbf{R}_{f}\left(\mathrm{SiO}_{2}\right.$, EtOAc/cyclohexane 1:1) $=0.21$

IR (ATR): $\tilde{v}\left[\mathrm{~cm}^{-1}\right]=3408$ (ss, br., OH), 2929 (s), 2853 (m), 1594 (m), 1483 (m), 1458 (s), 1406 (m), 1343 (m), 1322 (m), 1310 (m), 1249 (s), 1127 (s), 1093 (ss), 1004 (m), 886 (m), 833 (s), 775 (s), $668(\mathrm{w})$.

${ }^{1}$ H-NMR $\left(250 \mathrm{MHz}, \mathrm{CDCl}_{3}\right): \delta$ [ppm] $=-0.09$ (s, 3H, OSiMe), -0.07 (s, 3H, OSiMe), 0.82 (s, 9H, OSitBu), $1.68(\mathrm{~m}, 1 \mathrm{H}), 1.95(\mathrm{~m}, 1 \mathrm{H}), 2.36(\mathrm{~m}, 2 \mathrm{H}), 2.66(\mathrm{~m}, 2 \mathrm{H}), 3.63(\mathrm{~s}, 3 \mathrm{H}, \mathrm{OMe})$, $3.83(\mathrm{~s}, 6 \mathrm{H}, 2 \mathrm{OMe}), 3.96(\mathrm{~m}, 1 \mathrm{H}), 4.29(\mathrm{~m}, 1 \mathrm{H}), 4.36(\mathrm{~m}, 1 \mathrm{H}), 5.89\left(\mathrm{dd}, J_{1}=6 \mathrm{~Hz}, J_{2}=7 \mathrm{~Hz}\right.$, $1 \mathrm{H}, 12-\mathrm{H}), 6.10$ (d, J = $4 \mathrm{~Hz}, 1 \mathrm{H}, 8-\mathrm{H}), 6.43$ (s, 1H, 4-H).

${ }^{13}$ C-NMR (62.5 MHz, $\mathrm{CDCl}_{3}$ ): $\delta$ [ppm] = -5.09 (q, OSiMe), -4.98 (q, OSiMe), 18.23 (s, $\mathrm{OSiCMe}_{3}$ ), 25.83 (q, OSiCMe $), 29.65$ (t), 34.51 (t), 36.39 (t), 55.98 (q, OMe), 60.86 (q, 
OMe), 61.05 (q, OMe), 71.98 (d), 72.63 (d), 78.67 (d), 107.01 (d, C-4), 126.81 (s), 127.12 (d), 130.61 (d), 134.65 (s), 135.18 (s), 140.23 (s), 140.76 (s), 150.51 (s), 152.07.

MS (DIP-EI, $70 \mathrm{eV}): m / z(\%)=462$ (7) [M] ${ }^{+}, 405$ (19) [- $\left.\mathrm{C}_{4} \mathrm{H}_{9}\right], 387$ (24), 361 (12), 313 (21), 285 (22), 271 (18), 254 (12), 233 (11), 207 (23), 181 (28), 129 (17), 115 (14), 75 (88), 73 $(100)$.

HRMS (EI): calc. for $[\mathrm{M}]+{ }^{+}\left({ }^{12} \mathrm{C}_{25} \mathrm{H}_{38}{ }^{16} \mathrm{O}_{6}{ }^{28} \mathrm{Si}\right)$ :462.2438, found.: 462.244 .

$[\alpha]_{\mathrm{D}}=-81.3,[\alpha]_{546}=-96.2,[\alpha]_{405}=-196.5,\left(c=1.31, \mathrm{CHCl}_{3}, 20^{\circ} \mathrm{C}\right)$.

(7RS)-11-Amino-7-[[tert-butyl(dimethyl)silyl]oxy]-1,2,3-trimethoxy-6,7dihydrobenzo $[a]$ heptalen-10 $(5 \mathrm{H})$-one ( $\mathrm{rac}-17)$

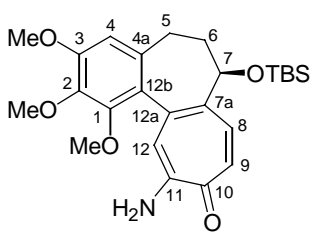

To a solution of tropone ( $r a c-14)(180 \mathrm{mg}, 407 \mu \mathrm{mol})$ in abs. EtOH $(8 \mathrm{~mL})$ under argon was added dropwise $\mathrm{N}_{2} \mathrm{H}_{4} \cdot \mathrm{H}_{2} \mathrm{O}(0.89 \mathrm{ml}, 18 \mathrm{mmol}, 45$ equiv. $)$ at $0{ }^{\circ} \mathrm{C}$. The solution was warmed to RT and stirred $4.5 \mathrm{~h}$. Solvent and liquid reagents were removed under vacuum. Radial chromotagraphy (EtOAc/cyclohexane 1:4+2\% $\mathrm{NEt}_{3}, 1: 3+2 \% \mathrm{NEt}_{3}$ ) afforded in the first fraction a yellow oil and in the second fraction a orange-yellow oil. Crystallisation of the second fraction in EtOAc/cyclohexane afforded $72 \mathrm{mg}$ (39\%) of orange-yellow needles.

$\mathbf{R}_{f}\left(\mathrm{SiO}_{2}\right.$, EtOAc/cyclohexane 1:3 + 2\% $\left.\mathrm{NEt}_{3}\right)=0.31$

mp.: $173{ }^{\circ} \mathrm{C}$ (EtOAc/cyclohexane)

IR (ATR): $\tilde{v}\left[\mathrm{~cm}^{-1}\right]=3419$ (w), 3288 (s, br.), 3187 (w), 2929 (m), 2853 (w), 1591 (s), 1523 (s), 1461 (m), 1433 (w), 1399 (w), 1359 (s), 1309 (w), 1249 (m), 1193 (m), 1123 (s), 1094 (ss), 1003 (m), 881 (s), 835 (s), 776 (m), 731 (m), 668 (w).

${ }^{1} \mathbf{H}$-NMR $\left(250 \mathrm{MHz}, \mathrm{CDCl}_{3}\right.$ ): $\delta$ [ppm] $=-0.15$ (s, 3H, OSiMe), -0.10 (s, 3H, OSiMe), 0.82 (s, 9H, OSitBu), 1.84 (m, 1H), 2.29 (m, 3H), 3.54 (s, 3H, OMe), 3.89 (s, 3H, OMe), 3.90 (s, 3H, OMe), 4.29 (m, 1H, 7-H), 5.75 (br. s, 2 NH), 6.52 (s, 1H, 4-H), 7.01 (s, 1H, 12-H), 7.25 $\left(\mathrm{d}, 1 \mathrm{H},{ }^{3} \mathrm{~J}=12.5 \mathrm{~Hz}\right), 7.83\left(\mathrm{~d}, 1 \mathrm{H},{ }^{3} J=12.5 \mathrm{~Hz}\right)$.

${ }^{13} \mathbf{C}-N M R\left(62.5 \mathrm{MHz}, \mathrm{CDCl}_{3}\right.$ ): $\delta$ [ppm] $=-5.10$ (q, OSiMe), -5.03 (q, OSiMe), 18.14 (s, OSiCMe ) $_{3} 25.75$ (q, OSiCMe $), 30.10$ (t), 41.83 (t), 55.95 (q, OMe), 60.86 (q, OMe), 61.23 (q, OMe), 71.76 (d, C-7), 107.12 (d, C-4), 117.31 (d), 126.35 (s), 130.12 (d), 133.28 (d), 135.75 (s), 137.71 (s), 140.78 (s), 141.13 (s), 150.50 (s), 153.43 (s), 154.04 (s), 175.88 (s, C-10). 
MS (DIP-EI, $70 \mathrm{eV}): \mathrm{m} / \mathrm{z}(\%)=457(21)[\mathrm{M}]^{+}, 426(36)[-\mathrm{OMe}], 400$ (12) $\left[-\mathrm{C}_{4} \mathrm{H}_{9}\right], 326(12)$, 298 (10), 207 (19), 75 (30), 73 (100).

HRMS (EI): calc. for $[\mathrm{M}]{ }^{+}\left({ }^{12} \mathrm{C}_{25} \mathrm{H}_{35}{ }^{14} \mathrm{~N}^{16} \mathrm{O}_{5}{ }^{28} \mathrm{Si}\right)$ : 457.2285, found.: 457.228.

(7RS)-9-Amino-7-[[tert-butyl(dimethyl)silyl]oxy]-1,2,3-trimethoxy-6,7dihydrobenzo[a]heptalen-10(5H)-one (rac-18)

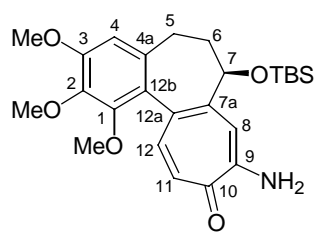

By the synthesis of (rac-17) from tropone ( $r a c-14)(180 \mathrm{mg}, 407 \mu \mathrm{mol})$, a yellow oil was obtained after chromatography in a first fraction. Crystallisation in EtOAc/cyclohexane afforded $78 \mathrm{mg}(42 \%)$ of yellow crystals.

$\mathbf{R}_{f}\left(\mathrm{SiO}_{2}\right.$, EtOAc/cyclohexane 1:3 + 2\% $\left.\mathrm{NEt}_{3}\right)=0.37$

mp.: $219-220{ }^{\circ} \mathrm{C}$ (destr.) (EtOAc/cyclohexane)

IR (ATR): $\tilde{v}\left[\mathrm{~cm}^{-1}\right]=3412(\mathrm{w}), 3285$ (s, br.), 2928 (m), 2852 (w), 1598 (s), 1522 (s), 1485 (m), 1444 (m), 1403 (m), 1341 (s), 1318 (m), 1258 (m), 1138 (m), 1095 (ss), 1004 (m), 885 (s), 835 (s), 777 (s), 668 (w).

${ }^{1}$ H-NMR (300 MHz, $\mathrm{CDCl}_{3}$ ): $\delta$ [ppm] $=-0.12$ (s, 3H, OSiMe), -0.08 (s, 3H, OSiMe), 0.84 $(\mathrm{s}, 9 \mathrm{H}, \mathrm{OSi} t \mathrm{Bu}), 1.88\left(\mathrm{~m}, 1 \mathrm{H}, 6-\mathrm{H}_{\mathrm{a}}\right), 2.31\left(\mathrm{~m}, 3 \mathrm{H}, 6-\mathrm{H}_{\mathrm{b}}\right.$ und 5- $\left.\mathrm{H}_{2}\right), 3.54(\mathrm{~s}, 3 \mathrm{H}, \mathrm{OMe}$ an $\mathrm{C}-1)$, 3.89 (s, 3H, OMe an C-3), 3.89 (s, 3H, OMe an C-2), 4.35 (m, 1H, 7-H), 5.88 (br. s, $2 \mathrm{NH}$ ), $6.51(\mathrm{~s}, 1 \mathrm{H}, 4-\mathrm{H}), 7.12\left(\mathrm{~d},{ }^{3} J=12.5 \mathrm{~Hz}, 11-\mathrm{H}\right), 7.38\left(\mathrm{~d},{ }^{3} \mathrm{~J}=12.5 \mathrm{~Hz}, 12-\mathrm{H}\right), 7.50(\mathrm{~s}, 8-\mathrm{H}) .{ }^{*}$

${ }^{13}$ C-NMR (75 MHz, $\mathrm{CDCl}_{3}$ ): $\delta$ [ppm] = -5.09 (q, OSiMe), -4.95 (q, OSiMe), 18.24 (s,

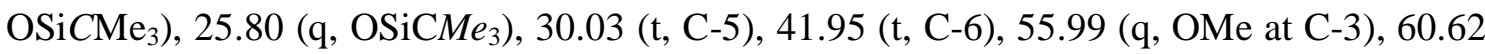
(q, OMe at C-1), 61.24 (q, OMe at C-2), 72.34 (d, C-7), 107.00 (d, C-4), 109.51 (d, C-8), 125.93 (s, C-12b), 128.70 (d, C-11), 129.14 (s, C-12a), 135.58 (s, C-4a), 140.73 (d, C-12), 141.16 (s, C-2), 149.90 (s, C-7a), 150.57 (s, C-1), 152.96 (s, C-3), 155.23 (s, C-9), 175.72 (s, C-10). ${ }^{*}$

MS (DIP-EI, $70 \mathrm{eV}): m / z(\%)=457(17)[\mathrm{M}]^{+}, 400(9)\left[-\mathrm{C}_{4} \mathrm{H}_{9}\right], 287$ (10), 167 (20), 149 (48), 126 (100), 97 (28), 83 (21), 71 (38).

HRMS (EI): calc. for $[\mathrm{M}]]^{+}\left({ }^{12} \mathrm{C}_{25} \mathrm{H}_{35}{ }^{14} \mathrm{~N}^{16} \mathrm{O}_{5}{ }^{28} \mathrm{Si}\right)$ : 457.2285 , found.: 457.229 .

*) Peaks in ${ }^{1} \mathrm{H}$-NMR- und ${ }^{13} \mathrm{C}$-NMR-spectra were assigned with the help of H,H-Cosy-, HMQC-, HMBC- und NOESY-spectra. 
(7RS)-11-Amino-7-hydroxy-1,2,3-trimethoxy-6,7-dihydrobenzo[a]heptalen-10(5H)-one $($ rac-19)

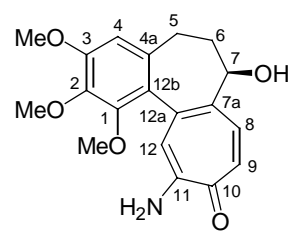

To a solution of silylether ( $r a c-17)(54 \mathrm{mg}, 0.12 \mathrm{mmol})$ in abs. THF $(1 \mathrm{~mL})$ under argon was added dropwise at $0^{\circ} \mathrm{C}$ a solution of TBAF $(0.35 \mathrm{~mL}, 0.35 \mathrm{mmol}, 2.9$ equiv., $1 \mathrm{M}$ in THF). After $45 \mathrm{~min}$, water $(5 \mathrm{~mL})$ was added and the mixture was extracted with $\mathrm{CH}_{2} \mathrm{Cl}_{2}(4 \times 4 \mathrm{~mL})$. The combined organic extracts were filtered in a Pasteur-pipette over a short bed of Celite and the solvent was removed under vacuum. The raw product was purified by radial chromatography (EtOAC/cyclohexane 5:1 + $1 \% \mathrm{NEt}_{3}$ ) to afford $39 \mathrm{mg}(96 \%)$ of a yellow oil.

$\mathbf{R}_{f}\left(\mathrm{SiO}_{2}\right.$, EtOAc/cyclohexane 10:1) $=0.13$

IR (ATR): $\tilde{v}\left[\mathrm{~cm}^{-1}\right]=3433$ (m), 3323 (s, br.), 3194 (m), 2924 (s), 2850 (w), 1707 (w), 1592 (ss), 1517 (s), 1490 (s), 1445 (ss), 1402 (m), 1345 (s), 1313 (m), 1234 (m), 1194 (m), 1123 (ss), $1089(\mathrm{~m}), 848(\mathrm{w}), 746(\mathrm{w})$.

${ }^{1}$ H-NMR $\left(250 \mathrm{MHz}, \mathrm{CDCl}_{3}\right): \delta[\mathrm{ppm}]=1.85(\mathrm{~m}, 1 \mathrm{H}), 2.35(\mathrm{~m}, 3 \mathrm{H}), 3.56(\mathrm{~s}, 3 \mathrm{H}, \mathrm{OMe}), 3.87$ (s, 3H, OMe), 3.88 (s, 3H, OMe), 4.41 (dd, $\left.J_{1}=5.5 \mathrm{~Hz}, J_{2}=10.5 \mathrm{~Hz}, 1 \mathrm{H}, 7-\mathrm{H}\right), 5.77$ (br. s, 2 $\mathrm{NH}), 6.53$ (s, 1H, 4-H), 7.00 (s, 1H, C-12), $\left.7.18^{*}\right)\left(\mathrm{d},{ }^{3} J=12.5 \mathrm{~Hz}, 1 \mathrm{H}\right), 7.84\left(\mathrm{~d},{ }^{3} J=12.5 \mathrm{~Hz}\right.$, $1 \mathrm{H})$.

${ }^{13}$ C-NMR (62.5 MHz, $\left.\mathrm{CDCl}_{3}\right): \delta$ [ppm] = $30.07(\mathrm{t}), 40.66(\mathrm{t}), 56.02(\mathrm{q}, \mathrm{OMe}), 61.00(\mathrm{q}$, OMe), 61.20 (q, OMe), 70.78 (d, C-7), 107.21 (d, C-4), 117.93 (d), 126.26 (s), 130.11 (d), 132.82 (d), 135.76 (s), 138.24 (s), 141.03 (s), 141.51 (s), 150.47 (s), 153.50 (s), 154.19 (s), 175.46 (s, C-10).

MS (DIP-EI, $70 \mathrm{eV}): m / z(\%)=343(100)[\mathrm{M}]^{+}, 325(12)\left[-\mathrm{H}_{2} \mathrm{O}\right], 312$ (34) [-OMe], 284 (10), 267 (17), 256 (10), 207 (40), 181 (20), 128 (9), 115 (13), 83 (13) 77 (14).

HRMS (EI): calc. for $[\mathrm{M}]+\left({ }^{+} \mathrm{C}_{19} \mathrm{H}_{21}{ }^{14} \mathrm{~N}^{16} \mathrm{O}_{5}\right): 343.1420$, found.: 343.142 .

*) The doublet-signal at $7.18 \mathrm{ppm}$ in ${ }^{1} \mathrm{H}-\mathrm{NMR}$-Spectrum is strongly dependent on the concentration. 
(7RS)-9-Amino-7-hydroxy-1,2,3-trimethoxy-6,7-dihydrobenzo[ $[a]$ heptalen-10(5H)-one $($ rac-20)

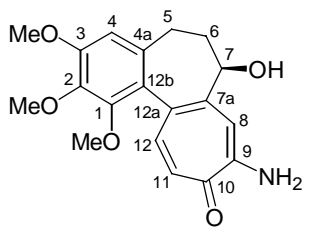

To a solution of silylether $(\mathrm{rac}-\mathbf{1 8})(51 \mathrm{mg}, 0.11 \mathrm{mmol})$ in abs. THF $(1 \mathrm{~mL})$ under argon was added dropwise at $0^{\circ} \mathrm{C}$ a solution of TBAF $(0.30 \mathrm{~mL}, 0.30 \mathrm{mmol}, 2.7$ equiv., $1 \mathrm{M}$ in THF). After $45 \mathrm{~min}$, water $(5 \mathrm{~mL})$ was added and the mixture was extracted with $\mathrm{CH}_{2} \mathrm{Cl}_{2}(4 \times 4 \mathrm{~mL})$. The combined organic extracts were filtered in a Pasteur-pipette over a short bed of Celite and the solvent was removed under vacuum. The raw product was purified by radial chromatography (EtOAC/Cyclohexane 5:1 + $2 \% \mathrm{NEt}_{3}, 10: 1+2 \% \mathrm{NEt}_{3}$ ) to afford $35 \mathrm{mg}(91$ $\%$ ) of a light yellow solid.

$\mathbf{R}_{f}\left(\mathrm{SiO}_{2}\right.$, EtOAc/cyclohexane 10:1 $\left.+2 \% \mathrm{NEt}_{3}\right)=0.14$

IR (ATR): $\tilde{v}\left[\mathrm{~cm}^{-1}\right]=3353(\mathrm{~s}), 3288(\mathrm{~m}), 3211(\mathrm{~m}), 2932(\mathrm{w}), 1643(\mathrm{w}), 1602$ (s), 1518 (s), 1476 (s), 1444 (ss), 1402 (m), 1338 (s), 1292 (m), 1195 (m), 1137 (s), 1097 (ss), 1054 (m), $857(\mathrm{~m}), 726(\mathrm{~s}), 648(\mathrm{w})$.

${ }^{1}$ H-NMR (250 MHz, DMSO-[D $]$ ): $\delta$ [ppm] = $1.72(\mathrm{~m}, 1 \mathrm{H}), 2.19(\mathrm{~m}, 2 \mathrm{H}), 2.46(\mathrm{~m}, 1 \mathrm{H}), 3.53$ (s, 3H, OMe), 3.75 (s, 3H, OMe), 3.82 (s, 3H, OMe), 4.19 (m, 1H, 7-H), 5.51 (d, $1 \mathrm{OH}), 6.74$ (s, 1H, 4-H), $6.84\left(\mathrm{~d},{ }^{3} \mathrm{~J}=12 \mathrm{~Hz}, 1 \mathrm{H}\right), 7.14\left(\mathrm{~d},{ }^{3} \mathrm{~J}=12 \mathrm{~Hz}, 1 \mathrm{H}\right), 7.44$ (br. s, $\left.2 \mathrm{NH}\right), 7.62$ (s, $1 \mathrm{H}, \mathrm{C}-8)$.

${ }^{13}$ C-NMR (62.5 MHz, DMSO-[D 6 ] $): \delta$ [ppm] = $\left.29.28(\mathrm{t}), 40.43^{*}\right)(\mathrm{t}), 55.73(\mathrm{q}, \mathrm{OMe}), 60.24$ (q, OMe), 60.44 (q, OMe), 69.97 (d, C-7), 107.29 (d), 107.82 (d), 125.82 (d), 125.91 (s), 126.47 (s), 135.11 (s), 139.18 (d), 140.35 (s), 149.77 (s), 150.98 (s), 152.22 (s), 156.89 (s), 174.20 (s, C-10).

MS (DIP-EI, $70 \mathrm{eV}): m / z(\%)=344$ (21), $343(100)[\mathrm{M}]^{+}, 315$ (15), 256 (12), 181 (52), 128 (10), 115 (10), 84 (12).

HRMS (EI): calc. für [M] ${ }^{+}\left({ }^{12} \mathrm{C}_{19} \mathrm{H}_{21}{ }^{14} \mathrm{~N}^{16} \mathrm{O}_{5}\right): 343.1420$, found.: 343.142 .

*) The signal at $40.43 \mathrm{ppm}$ in ${ }^{13} \mathrm{C}$-NMR-spectrum was overlapped by the solvent signal and was determined using a DEPT-spectrum. 


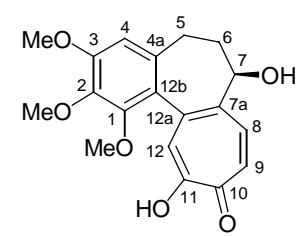

A solution of aminotropone ( $r a c-19)(38 \mathrm{mg}, 0.11 \mathrm{mmol})$ in a mixture of EtOH $(2 \mathrm{~mL})$ and aq. $\mathrm{KOH}(2 \mathrm{~mL}, 2 \mathrm{~N})$ was heated overnight at $110{ }^{\circ} \mathrm{C}$ under argon. The solution was then cooled to $0^{\circ} \mathrm{C}$, neutralized with $\mathrm{HCL} 1 \mathrm{~N}$ and extracted with $\mathrm{CH}_{2} \mathrm{Cl}_{2}(3 \times 4 \mathrm{~mL})$. The combined organic extracts were filtered in a Pasteur-pipette over a short bed of Celite. Removal of the solvent under vacuum afforded $32 \mathrm{mg}(82 \%)$ of a yellow oil, containing $~ 5 \%$ impurities (according to NMR).

IR (ATR): $\tilde{v}\left[\mathrm{~cm}^{-1}\right]=3378$ (ss, br.), 3215 (s, br.), 2934 (s), 2853 (w), 1591 (ss), 1540 (m), 1456 (ss), 1399 (m), 1345 (m), 1316 (m), 1266 (s), 1236 (s), 1193 (m), 1130 (s), 1109 (s), 1088 (s), 1050 (m), 997 (m), $970(\mathrm{w}), 919(\mathrm{w}), 850$ (m), 733 (s).

${ }^{1}$ H-NMR (300 MHz, DMSO-[D 6 ): $\delta$ [ppm] = $1.69(\mathrm{~m}, 1 \mathrm{H}), 2.20(\mathrm{~m}, 2 \mathrm{H}), 2.45(\mathrm{~m}, 1 \mathrm{H}), 3.60$ (s, 3H, OMe), $3.78(\mathrm{~s}, 3 \mathrm{H}, \mathrm{OMe}), 3.85(\mathrm{~s}, 3 \mathrm{H}, \mathrm{OMe}), 4.16\left(\mathrm{dd}, J_{1}=6.5, J_{2}=10.5 \mathrm{~Hz}, 1 \mathrm{H}\right.$, 7-H), 5.51 (br. s, $1 \mathrm{OH}), 6.79$ (s, 1H, 4-H), 7.15 (s, 1H, 12-H), 7.33 (d, $\left.{ }^{3} J=12 \mathrm{~Hz}, 1 \mathrm{H}, 9-\mathrm{H}\right)$, $7.91\left(\mathrm{~d},{ }^{3} \mathrm{~J}=12 \mathrm{~Hz}, 1 \mathrm{H}, 8-\mathrm{H}\right)$.

${ }^{13}$ C-NMR (75 MHz, DMSO-[D 6 ) : $\delta[\mathrm{ppm}]=29.10(\mathrm{t}), 40.19(\mathrm{t}), 55.78(\mathrm{q}, \mathrm{OMe}), 60.49(\mathrm{q}$, OMe), 60.72 (q, OMe), 69.40 (d, C-7), 107.45 (d, C-4), 124.98 (s), 125.36 (d), 126.26 (d), 132.84 (d), 135.17 (s), 140.35 (s), 141.42 (s), 142.69 (s), 149.80 (s), 153.38 (s), 166.27 (s, C-11), 172.49 (s, C-10).

MS (DIP-EI, $70 \mathrm{eV}): m / z(\%)=344(34)[\mathrm{M}]^{+}, 298$ (12) [- $\left.\mathrm{H}_{2} \mathrm{O}-\mathrm{CO}\right], 207$ (9), 181 (16), 167 (16), 149 (36), 126 (20), 111 (32), 97 (48), 84 (75), 71 (53).

HRMS (EI): calc. for $[\mathrm{M}]{ }^{+}\left({ }^{12} \mathrm{C}_{19} \mathrm{H}_{20}{ }^{16} \mathrm{O}_{6}\right)$ : 344.1260 , found.: 344.126 .

(7RS)-7,9-Dihydroxy-1,2,3-trimethoxy-6,7-dihydrobenzo[a]heptalen-10(5H)-one (rac22) (( \pm )-7-Hydroxydesacetamidocolchicein)

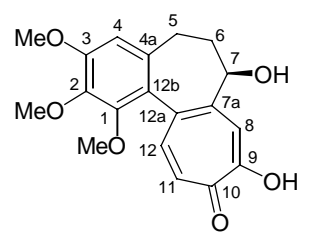


A solution of aminotropone (rac-20) $(31 \mathrm{mg}, 90 \mu \mathrm{mol})$ in a mixture of EtOH $(2 \mathrm{~mL})$ and aq. $\mathrm{KOH}(2 \mathrm{~mL}, 2 \mathrm{~N})$ was heated overnight at $110{ }^{\circ} \mathrm{C}$ under argon. The solution was then cooled to $0^{\circ} \mathrm{C}$, neutralized with $\mathrm{HCL} 1 \mathrm{~N}$ and extracted with $\mathrm{CH}_{2} \mathrm{Cl}_{2}(3 \times 4 \mathrm{~mL})$. The combined organic extracts were filtered in a Pasteur-pipette over a short bed of Celite. Removal of the solvent under vacuum afforded $23 \mathrm{mg}(74 \%)$ of a yellow oil.

IR (ATR): $\tilde{v}\left[\mathrm{~cm}^{-1}\right]=3340$ (ss, br., OH), 3215 (s, br.), 2922 (s), 2850 (w), 1596 (ss), 1545 (m), 1487 (s), 1453 (m), 1401 (m), 1344 (m), 1318 (m), 1273 (s), 1231 (s), 1194 (m), 1137 (s), 1092 (ss), 1001 (m), 923 (m), 857 (w), 753 (w).

${ }^{1}$ H-NMR $\left(250 \mathrm{MHz}, \mathrm{CDCl}_{3}\right): \delta[\mathrm{ppm}]=1.84(\mathrm{~m}, 1 \mathrm{H}), 2.28(\mathrm{~m}, 1 \mathrm{H}), 2.40(\mathrm{~m}, 2 \mathrm{H}), 3.68(\mathrm{~s}$, 3H, OMe), 3.85 (s, 3H, OMe), 3.88 (s, 3H, OMe), 4.44 (m, 1H, 7-H), 5.57 (br. s, $1 \mathrm{OH}), 6.52$ (s, 1H, 4-H), 7.34 (d, $\left.{ }^{3} J=12 \mathrm{~Hz}, 1 \mathrm{H}\right), 7.43$ (s, 1H, C-12), 8.02 (d, $\left.{ }^{3} J=12 \mathrm{~Hz}, 1 \mathrm{H}\right)$.

${ }^{13}$ C-NMR (75 MHz, DMSO-[D 6 ) : $\delta[\mathrm{ppm}]=29.05(\mathrm{t}), 40.34(\mathrm{t}), 55.77(\mathrm{q}, \mathrm{OMe}), 60.47(\mathrm{q}$, OMe), 60.54 (q, OMe), 69.84 (d, C-7), 107.42 (d, C-4), 118.10 (d), 124.33 (d), 124.81 (s), 132.94 (s), 135.11 (s), 140.16 (d), 140.35 (s), 149.82 (s), 151.84 (s), 152.94 (s), 168.18 (s), $172.03(\mathrm{~s})$.

MS (DIP-EI, $70 \mathrm{eV}): \mathrm{m} / \mathrm{z}(\%)=344(100)[\mathrm{M}]^{+}, 316(20)$ [-CO], 257 (15), 197 (10), 181 (60), 169 (10), 152 (10), 128 (13), 115 (20), 99 (13), 77 (15), 71 (13), 69 (16).

HRMS (EI): calc. for $[\mathrm{M}]{ }^{+}\left({ }^{12} \mathrm{C}_{19} \mathrm{H}_{20}{ }^{16} \mathrm{O}_{6}\right): 344.1260$, found.: 344.125 .

\section{(7R)-7-[[tert-Butyl(dimethyl)silyl]oxy]-1,2,3,10-tetramethoxy-6,7-} dihydrobenzo[ $[a]$ heptalen-9(5H)-one $(24)$

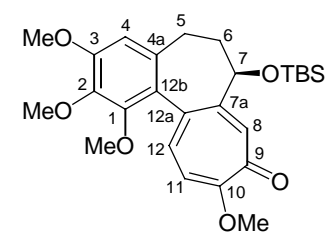

To a solution of DMSO (0.70 mL, $9.8 \mathrm{mmol}, 36$ equiv.) in $\mathrm{CH}_{2} \mathrm{Cl}_{2}$ (35 mL) under argon was added dropwise at $-60^{\circ} \mathrm{C}$ TFAA $(1.10 \mathrm{~mL}, 7.9 \mathrm{mmol}, 29$ equiv.). After $10 \mathrm{~min}$, a solution of diol (16) $(125 \mathrm{mg}, 270 \mu \mathrm{mol})$ in $\mathrm{CH}_{2} \mathrm{Cl}_{2}(5 \mathrm{~mL})$ was added and the resulting solution was stirred $1.5 \mathrm{~h}$ at $-60{ }^{\circ} \mathrm{C}$. $\mathrm{NEt}_{3}(2.73 \mathrm{~mL}, 19.7 \mathrm{mmol}, 73$ equiv.) was added and the solution was allowed to warm to RT overnight. $\mathrm{HCl} 0,1 \mathrm{~N}$ was then added to the pale-orange resulting solution and the phases were separated. The aqueous phase was extracted $\mathrm{CH}_{2} \mathrm{Cl}_{2}(2 \times 10 \mathrm{~mL})$ and the combined organic extracts were filtrated through a short bed of Celite. The solvent was removed under vacuum and the residue was solubilized in $\mathrm{MeOH}(15 \mathrm{~mL})$. To this 
solution, $\mathrm{CH}_{2} \mathrm{~N}_{2}\left(8 \mathrm{~mL}, 2.4 \mathrm{mmol}, 9\right.$ equiv., $0.3 \mathrm{M}$ in $\mathrm{Et}_{2} \mathrm{O}$ ) was added at $0{ }^{\circ} \mathrm{C}$. The resulting mixture was stirred $1 \mathrm{~h}$. Silica was then added until no more gas evolution was observed. After filtartion, the solvent was removed under vacuum. The raw product was purified by flash-chromatography (EtOAc/cyclohexane 1:2, 1:1, 2:1, 3:1) to afford $38 \mathrm{mg}$ of a yellow oil in a first fraction, $20.5 \mathrm{mg}$ of a colorless oil in a second fraction, $14.2 \mathrm{mg}$ of a mixed fraction and $26.8 \mathrm{mg}$ of a last fraction. Radial chromatography (EtOAc/cyclohexane 1:2,1:1) of the mixed fraction and of the last fraction together afforded $25.5 \mathrm{mg}(20 \%)$ of a yellow oil (the less polar compound).

$\mathbf{R}_{f}\left(\mathrm{SiO}_{2}, \mathrm{EtOAc} / n\right.$-hexane $\left.1: 2\right)=0.20$

IR (ATR): $\tilde{v}\left[\mathrm{~cm}^{-1}\right]=2928$ (s), 2853 (w), 1616 (m), 1586 (ss), 1486 (m), 1458 (s), 1343 (m), 1317 (m), 1248 (ss), 1193 (w), 1137 (m), 1118 (m), 1096 (ss), 1005 (m), 889 (m), 836 (ss), $778(\mathrm{~m}), 671(\mathrm{w})$.

${ }^{1} \mathbf{H}-\mathbf{N M R}\left(300 \mathrm{MHz}, \mathrm{CDCl}_{3}\right): \delta[\mathrm{ppm}]=-0.09$ (s, 6H, OSiMe $), 0.83$ (s, 9H, OSi $\left.t \mathrm{Bu}\right), 1.80$ $\left(\mathrm{m}, 1 \mathrm{H}, 6-\mathrm{H}_{\mathrm{a}}\right), 2.21\left(\mathrm{~m}, 1 \mathrm{H}, 6-\mathrm{H}_{\mathrm{b}}\right), 2.39\left(\mathrm{~m}, 2 \mathrm{H}, 5-\mathrm{H}_{2}\right), 3.57(\mathrm{~s}, 3 \mathrm{H}, \mathrm{OMe}$ an $\mathrm{C}-1), 3.912(\mathrm{~s}$, $3 \mathrm{H}, \mathrm{OMe}$ at C-3), 3.917 (s, 3H, OMe at C-2), 3.97 (s, 3H, OMe at C-9), 4.32 (dd, $J_{1}=7 \mathrm{~Hz}$, $\left.J_{2}=10 \mathrm{~Hz}, 1 \mathrm{H}, 7-\mathrm{H}\right), 6.52(\mathrm{~s}, 1 \mathrm{H}, 4-\mathrm{H}), 6.76\left(\mathrm{~d},{ }^{3} J=11 \mathrm{~Hz}, 1 \mathrm{H}, 11-\mathrm{H}\right), 7.19\left(\mathrm{~d},{ }^{3} J=11 \mathrm{~Hz}\right.$, $1 \mathrm{H}, 12-\mathrm{H}), 7.81$ (s, 1H, 8-H). ${ }^{*}$

${ }^{13}$ C-NMR $\left(75 \mathrm{MHz}, \mathrm{CDCl}_{3}\right): \delta[\mathrm{ppm}]=-5.01\left(\mathrm{q}, \mathrm{OSiMe}_{2}\right), 18.24\left(\mathrm{~s}, \mathrm{OSiCMe}_{3}\right), 25.82(\mathrm{q}$, OSiCMe ), 29.99 (t, C-5), 40.46 (t, C-6), 55.99 (q, OMe at C-3), 56.17 (q, OMe at C-10), 60.78 (q, OMe at C-1), 61.22 (q, OMe at C-2), 72.12 (d, C-7), 107.02 (d, C-4), 111.53 (d, C-11), 125.08 (s, C-12b), 132.21 (d, C-8), 134.32 (d, C-12), 134.71 (s, C-12a), 135.22 (s, C-4a), 141.19 (s, C-2), 150.75 (s, C-1), 152.29 (s, C-7a), 153.27 (s, C-3), 164.02 (s, C-10), 179.65 (s, C-9). ${ }^{*}$

MS (DIP-EI, $70 \mathrm{eV}): \mathrm{m} / z(\%)=472(43)[\mathrm{M}]^{+}, 415(65)\left[-\mathrm{C}_{4} \mathrm{H}_{9}\right], 387(62)\left[-\mathrm{C}_{4} \mathrm{H}_{9}-\mathrm{CO}\right], 372$ (27), 356 (17), 341 (10), 313 (16), 282 (16), 219 (12), 209 (24), 97 (17), 89 (50), 75 (68), 73 (100).

HRMS (EI): calc. for $[\mathrm{M}]]^{+}\left({ }^{12} \mathrm{C}_{26} \mathrm{H}_{36}{ }^{16} \mathrm{O}_{6}{ }^{28} \mathrm{Si}\right): 472.2281$, found.: 472.228 .

$[\alpha]_{\mathbf{D}}=+137.8,[\alpha]_{546}=+182.1\left(c=0.95, \mathrm{CHCl}_{3}, 20^{\circ} \mathrm{C}\right)$.

*) Peaks in ${ }^{1} \mathrm{H}-\mathrm{NMR}$ - und ${ }^{13} \mathrm{C}$-NMR-spectra were assigned with the help of H,H-Cosy-, HMQC-, HMBC- und NOESY-spectra.

(7R)-7-[[tert-Butyl(dimethyl)silyl]oxy]-1,2,3,9-tetramethoxy-6,7dihydrobenzo $[a]$ heptalen-10(5H)-one $(25)$ 


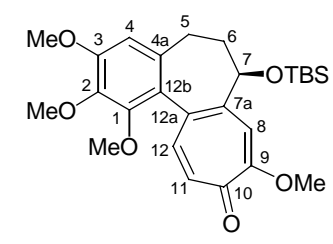

The second fraction obtained from the precedent synthesis of $\mathbf{2 4}$ and the more polar fraction obtained after chromatography of the mixed fraction afforded together $28.1 \mathrm{mg}(22 \%)$ of a colorless oil.

$\mathbf{R}_{f}\left(\mathrm{SiO}_{2}, \mathrm{EtOAc} / n\right.$-hexane $\left.1: 2\right)=0.27$

IR (ATR): $\tilde{v}\left[\mathrm{~cm}^{-1}\right]=2928(\mathrm{~m}), 2853(\mathrm{w}), 2148(\mathrm{w}), 1619(\mathrm{~s}), 1575$ (ss), $1488(\mathrm{~m}), 1458$ (s), 1402 (m), 1343 (m), 1320 (m), 1254 (s), 1191 (w), 1160 (m), 1139 (s), 1096 (ss), 1004 (m), $893(\mathrm{~m}), 836(\mathrm{ss}), 778(\mathrm{~m}), 671(\mathrm{w})$.

${ }^{1}$ H-NMR (300 MHz, $\mathrm{CDCl}_{3}$ ): $\delta$ [ppm] $=-0.10$ (s, 3H, OSiMe), -0.07 (s, 3H, OSiMe), 0.84 (s, 9H, OSitBu), $1.98\left(\mathrm{~m}, 1 \mathrm{H}, 6-\mathrm{H}_{\mathrm{a}}\right), 2.30\left(\mathrm{~m}, 1 \mathrm{H}, 6-\mathrm{H}_{\mathrm{b}}\right), 2.35\left(\mathrm{~m}, 2 \mathrm{H}, 5-\mathrm{H}_{2}\right), 3.59(\mathrm{~s}, 3 \mathrm{H}$, OMe at C-1), 3.891 (s, 3H, OMe at C-2), 3.894 (s, 3H, OMe at C-3), 3.99 (s, 3H, OMe at C-9), 4.39 (m, 1H, 7-H), 6.53 (s, 1H, 4-H), 7.15 (d, $\left.{ }^{3} J=12.5 \mathrm{~Hz}, 1 \mathrm{H}, 11-\mathrm{H}\right), 7.34$ (d, $\left.\left.{ }^{3} J=12.5 \mathrm{~Hz}, 1 \mathrm{H}, 12-\mathrm{H}\right), 7.55(\mathrm{~s}, 1 \mathrm{H}, 8-\mathrm{H}){ }^{*}\right)$

${ }^{13}$ C-NMR (75 MHz, $\left.\mathrm{CDCl}_{3}\right): \delta[\mathrm{ppm}]=-4.97$ (q, OSiMe), -4.91 (q, OSiMe), 18.12 (s, $\mathrm{OSiCMe}_{3}$ ), 25.77 (q, OSiCMe 3 ), 29.96 (t, C-5), 42.21 (t, C-6), 56.01 **) (q, OMe an C-3), $56.06^{* *}$ (q, OMe an C-9), 60.80 (q, OMe an C-1), 61.23 (q, OMe an C-2), 72.30 (d, C-7), 107.12 (d, C-4), 110.16 (d, C-8), 125.11 (s, C-12b), 132.61 (s, C-12a), 133.85 (d, C-11), 135.69 (s, C-4a), 141.00 (d, C-12), 141.18 (s, C-2), 146.03 (s, C-7a), 150.57 (s, C-1), 153.52 (s, C-3), 163.74 (s, C-9), 179.60 (s, C-10). ${ }^{*}$

MS (DIP-EI, $70 \mathrm{eV}): m / z(\%)=472(26)[\mathrm{M}]^{+}, 415(49)\left[-\mathrm{C}_{4} \mathrm{H}_{9}\right], 387(27)\left[-\mathrm{C}_{4} \mathrm{H}_{9}-\mathrm{CO}\right], 372$ (11), 341 (10), 313 (12), 285 (10), 97 (13), 83 (14), 75 (42), 73 (100).

HRMS (EI): calc. for $[\mathrm{M}]]^{+}\left({ }^{12} \mathrm{C}_{26} \mathrm{H}_{36}{ }^{16} \mathrm{O}_{6}{ }^{28} \mathrm{Si}\right): 472.2281$, found.: 472.228 .

$[\alpha]_{\mathrm{D}}=+231.6,[\alpha]_{546}=+313.6\left(c=0.80, \mathrm{CHCl}_{3}, 20^{\circ} \mathrm{C}\right)$.

*) Peaks in ${ }^{1} \mathrm{H}-\mathrm{NMR}$ - und ${ }^{13} \mathrm{C}$-NMR-spectra were assigned with the help of $\mathrm{H}, \mathrm{H}-\mathrm{Cosy}-$, HMQC-, HMBC- und NOESY-spectra.

**) The assignments of the signals at $56.01 \mathrm{ppm}$ und $56.06 \mathrm{ppm}$ in ${ }^{13} \mathrm{C}-\mathrm{NMR}$-spectra could be exhanged.

(7R)-7-Hydroxy-1,2,3,10-tetramethoxy-6,7-dihydrobenzo[a]heptalen-9(5H)-one (26) (7-Hydroxydesacetamidocolchicine) 


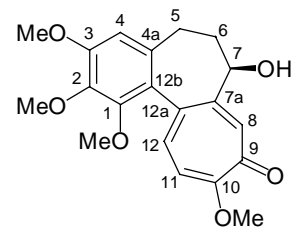

To a solution of silylether (24) $(20 \mathrm{mg}, 42 \mu \mathrm{mol})$ in abs. THF $(2 \mathrm{~mL})$, HF.Py $(0.50 \mathrm{~mL}, 1 \mathrm{M}$ in THF) was added at $0{ }^{\circ} \mathrm{C}$. After 30 min the solution was warmed to RT and HF.Py $(1.0 \mathrm{~mL}$, $1 \mathrm{M}$ in THF) was once more added. After $2 \mathrm{~h}$, saturated $\mathrm{NH}_{4} \mathrm{Cl}(5 \mathrm{~mL})$ was added and the solution was extracted with $\mathrm{CH}_{2} \mathrm{Cl}_{2}(3 \times 4 \mathrm{~mL})$. The combined organic extracts were washed with $\mathrm{HCl} 0.1 \mathrm{~N}(5 \mathrm{~mL})$, filtrated over a short bed of Celite and the solvent was removed under vacuum. Purification by radial chromatography on (EtOAc/cyclohexane 15:1, EtOAc, $\left.\mathrm{CHCl}_{3} / \mathrm{MeOH} 20: 1\right)$ afforded $13 \mathrm{mg}(86 \%)$ of a colorless oil.

$\mathbf{R}_{f}\left(\mathrm{SiO}_{2}, \mathrm{CHCl}_{3} / \mathrm{MeOH} 20: 1\right)=0.10$

IR (ATR): $\tilde{v}\left[\mathrm{~cm}^{-1}\right]=3363$ (s, br.), 2921 (s), 2829 (m), 1612 (m), 1586 (s), 1555 (ss), 1486 (m), 1459 (m), 1344 (m), 1317 (m), 1250 (ss), 1192 (w), 1137 (m), 1092 (ss), 1013 (w), 1000 (w), $921(\mathrm{w}), 842(\mathrm{w})$.

${ }^{1}$ H-NMR (250 MHz, $\left.\mathrm{CDCl}_{3}\right): \delta[\mathrm{ppm}]=1.80(\mathrm{~m}, 1 \mathrm{H}), 1.96(\mathrm{~d}, 1 \mathrm{OH}), 2.43(\mathrm{~m}, 3 \mathrm{H}), 3.57(\mathrm{~s}$, 3H, OMe), 3.89 (s, 3H, OMe), 3.90 (s, 1H, OMe), 3.94 (s, 3H, OMe), 4.47 (m, 1H, 7-H), 6.54 (s, 1H, 4-H), $6.79\left(\mathrm{~d},{ }^{3} \mathrm{~J}=11 \mathrm{~Hz}, 1 \mathrm{H}\right), 7.18\left(\mathrm{~d},{ }^{3} \mathrm{~J}=11 \mathrm{~Hz}, 1 \mathrm{H}\right), 7.97$ (s, 1H, 8-H).

${ }^{13}$ C-NMR (62.5 MHz, $\left.\mathrm{CDCl}_{3}\right): \delta[\mathrm{ppm}]=29.85(\mathrm{t}), 39.0(\mathrm{t}), 56.07$ (q, OMe), $56.20(\mathrm{q}, \mathrm{OMe})$, 60.96 (q, OMe), 61.17 (q, OMe), 71.23 (d, C-7), 107.08 (d, C-4), 112.20 (d), 124.91 (s), 131.35 (d), 134.87 (d), 135.11 (s), 135.50 (s), 141.14 (s), 150.71 (s), 153.40 (s), 153.47 (s), 163.90 (s, C-10), 179.56 (s, C-9).

MS (DIP-EI, $70 \mathrm{eV}): m / z(\%)=358$ (56) $\left[\mathrm{M}^{+}\right], 330$ (46) [-CO], 271 (21), 255 (12), 241 (11), 181 (100), 152 (14), 128 (16), $115(19), 55$ (12).

HRMS (EI): calc. for $[\mathrm{M}]]^{+}\left({ }^{12} \mathrm{C}_{20} \mathrm{H}_{22}{ }^{16} \mathrm{O}_{6}\right): 358.1416$, found: 358.141 .

$[\alpha]_{\mathrm{D}}=+109,[\alpha]_{546}=+162\left(c=1.05, \mathrm{CHCl}_{3}, 20^{\circ} \mathrm{C}\right)$.

(7R)-7-Hydroxy-1,2,3,9-tetramethoxy-6,7-dihydrobenzo[ $[a]$ heptalen-10(5H)-one (27) (7-Hydroxydesacetamidoisocolchicine)

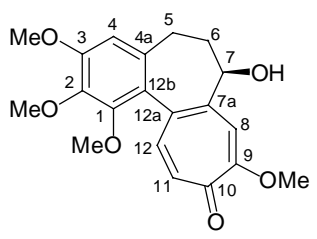


To a solution of silylether (25) $(45 \mathrm{mg}, 95 \mu \mathrm{mol})$ in abs. THF $(5 \mathrm{~mL})$, HF.Py $(0.50 \mathrm{~mL}, 1 \mathrm{M}$ in THF) was added at $0{ }^{\circ} \mathrm{C}$. After 30 min the solution was warmed to RT and HF.Py $(1.0 \mathrm{~mL}$, $1 \mathrm{M}$ in THF) was once more added. After $2 \mathrm{~h}$, saturated $\mathrm{NH}_{4} \mathrm{Cl}(5 \mathrm{~mL})$ was added and the solution was extracted with $\mathrm{CH}_{2} \mathrm{Cl}_{2}(3 \times 4 \mathrm{~mL})$. The combined organic extracts were washed with $\mathrm{HCl} 0.1 \mathrm{~N}(5 \mathrm{~mL})$, filtrated through a short bed of Celite and the solvent was removed under vacuum. Purification by radial chromatography (EtOAc/cyclohexane 20:1, EtOAc, $\mathrm{CHCl}_{3} / \mathrm{MeOH}$ 10:0.4) afforded $34 \mathrm{mg}(98 \%)$ of a pale yellow powder.

$\mathbf{R}_{f}\left(\mathrm{SiO}_{2}, \mathrm{CHCl}_{3} / \mathrm{MeOH}\right.$ 10:0.6) $=0.29$

IR (ATR): $\tilde{v}\left[\mathrm{~cm}^{-1}\right]=3347$ (s, br.), 2929 (s), 2850 (m), 1606 (m), 1555 (ss), 1487 (m), 1453 (m), 1402 (m), 1344 (m), 1318 (m), 1255 (m), 1234 (w), 1192 (w), 1161 (w), 1138 (m), 1093 (ss), 1048 (w), 999 (w), 977 (w), 918 (w), 851 (w).

${ }^{1}$ H-NMR $\left(300 \mathrm{MHz}, \mathrm{CDCl}_{3}\right): \delta[\mathrm{ppm}]=1.98(\mathrm{~m}, 1 \mathrm{H}), 2.43(\mathrm{~m}, 4 \mathrm{H}), 3.65(\mathrm{~s}, 3 \mathrm{H}, \mathrm{OMe})$, 3.865 (s, 3H, OMe), 3.878 (s, 1H, OMe), 3.981 (s, 3H, OMe), 4.49 (m, 1H, 7-H), 6.53 (s, 1H, $4-\mathrm{H}), 7.08\left(\mathrm{~d},{ }^{3} \mathrm{~J}=12.5 \mathrm{~Hz}, 1 \mathrm{H}\right), 7.30\left(\mathrm{~d},{ }^{3} \mathrm{~J}=12.5 \mathrm{~Hz}, 1 \mathrm{H}\right), 7.62(\mathrm{~s}, 1 \mathrm{H}, 8-\mathrm{H})$.

${ }^{13}$ C-NMR $\left(75 \mathrm{MHz}, \mathrm{CDCl}_{3}\right): \delta[\mathrm{ppm}]=29.93(\mathrm{t}, \mathrm{C}-5), 41.04$ (t, C-6), 56.04 (q, OMe), 56.25 (q, OMe), 61.01 (q, OMe), 61.13 (q, OMe), 71.29 (d, C-7), 107.17 (d, C-4), 110.60 (d, C-8), 124.92 (s, C-12b), 131.30, 133.46, 133.50, 135.71, 141.03 (s, C-2), 141.44 (s, C-7a), 150.55 (s, C-1), 153.61 (s, C-3), 163.79 (s, C-9), 179.35 (s, C-10).

MS (DIP-EI, $70 \mathrm{eV}): \mathrm{m} / z(\%)=358$ (56) $\left[\mathrm{M}^{+}\right], 330$ (46) [-CO], 271 (21), 255 (12), 241 (11), 181 (100), 152 (14), 128 (16), 115(19), 83 (12).

HRMS (EI): calc. for $[\mathrm{M}]^{+}\left({ }^{12} \mathrm{C}_{20} \mathrm{H}_{22}{ }^{16} \mathrm{O}_{6}\right): 358.1416$, found: 358.141 .

$[\alpha]_{\mathrm{D}}=+170,[\alpha]_{546}=+239\left(c=1.037, \mathrm{CHCl}_{3}, 20^{\circ} \mathrm{C}\right)$.

(7RS)-Methanesulfonic acid 1,2,3,10-tetramethoxy-9-oxo-5,6,7-trihydro-benzo[a]heptalen-7-yl ester (rac-28)

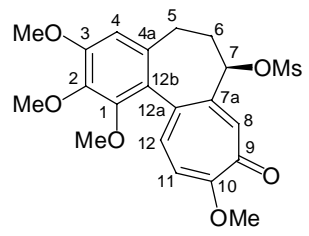

To a solution of alcohol (rac-26) $(316 \mathrm{mg}, 0.88 \mathrm{mmol})$ in abs. $\mathrm{CH}_{2} \mathrm{Cl}_{2}(20 \mathrm{~mL})$ under argon were sequently added $\mathrm{NEt}_{3}(0.8 \mathrm{~mL}, 5.73 \mathrm{mmol}, 6.5$ equiv. $)$ and freshly distilled $\mathrm{MsCl}(0.11$ $\mathrm{mL}, 1.41 \mathrm{mmol}, 1.4$ equiv.) at $0^{\circ} \mathrm{C}$. After $2 \mathrm{~h}$, ice-water $(5 \mathrm{~mL})$ was added and the solution was extracted with $\mathrm{CH}_{2} \mathrm{Cl}_{2}(3 \times 10 \mathrm{~mL})$. The combined organic extracts were washed with $\mathrm{HCl}$ 
$1 \mathrm{~N}(10 \mathrm{~mL})$, saturated $\mathrm{NaHCO}_{3}(10 \mathrm{~mL})$ and brine $(10 \mathrm{~mL})$, dried over $\mathrm{Na}_{2} \mathrm{SO}_{4}$ and filtered. The solvent was removed in vacuum and the raw product was purified by flash chromatography $\left(\mathrm{CH}_{2} \mathrm{Cl}_{2}\right.$ /acetone 1:1) to afford $317.1 \mathrm{mg}(82 \%)$ of a yellow oil.

$\mathbf{R}_{f}\left(\mathrm{SiO}_{2}, \mathrm{CH}_{2} \mathrm{Cl}_{2} /\right.$ acetone $\left.1: 1\right)=0.32$

IR (ATR): $\tilde{v}\left[\mathrm{~cm}^{-1}\right]=2934$ (s, br.), 2832 (m), 2235 (m), 1617 (m), 1566 (ss), 1485 (m), 1454 (m), 1431 (w), 1398 (m), 1344 (ss), 1319 (m), 1282 (w), 1248 (ss), 1172 (ss), 1136 (ss), 1087 (ss), 1064 (w), 1021 (m), 983 (m), 954 (m), 910 (m), 877 (m), 824 (m), 725 (ss), 643 (m).

${ }^{1}$ H-NMR $\left(300 \mathrm{MHz}, \mathrm{CDCl}_{3}\right): \delta[\mathrm{ppm}]=2.0(\mathrm{~m}, 1 \mathrm{H}), 2.45(\mathrm{~m}, 3 \mathrm{H}), 2.94$ (s, 3H, OMs), 3.54 (s, 3H, OMe), 3.84 (s, 3H, OMe), 3.85 (s, 1H, OMe), 3.94 (s, 3H, OMe), 5.18 (m, 1H, 7-H), $6.48(\mathrm{~s}, 1 \mathrm{H}, 4-\mathrm{H}), 6.78\left(\mathrm{~d},{ }^{3} \mathrm{~J}=10.8 \mathrm{~Hz}, 1 \mathrm{H}\right), 7.20\left(\mathrm{~d},{ }^{3} \mathrm{~J}=10.8 \mathrm{~Hz}, 1 \mathrm{H}\right), 7.55(\mathrm{~s}, 1 \mathrm{H}, 8-\mathrm{H})$.

${ }^{13}$ C-NMR $\left(75 \mathrm{MHz}, \mathrm{CDCl}_{3}\right): \delta[\mathrm{ppm}]=29.13(\mathrm{t}, \mathrm{C}-5), 37.59(\mathrm{t}, \mathrm{C}-6), 38.79\left(\mathrm{q}, \mathrm{SO}_{2} \mathrm{Me}\right)$, 55.89 (q, OMe), 56.31 (q, OMe), 61.05 (2×q, OMe), 78.27 (d, C-7), 107.39 (d, C-4), 112.13 (d, C-11), 123.98 (s), 130.98 (d, C-8), 133.55 (s), 133.85 (s), 135.60 (d, C-12), 141.48 (s), 146.55 (s), 150.85 (s), 153.69 (s), 164.24 (s, C-10), 178.73 (s, C-9).

MS (DIP-EI, $70 \mathrm{eV}): m / z(\%)=436$ (26) [M+], 312 (100), 297 (28), 281 (25), 269 (10), 254 (15), 79 (33).

HRMS (EI): calc. for $[\mathrm{M}]{ }^{+}\left({ }^{12} \mathrm{C}_{21} \mathrm{H}_{24}{ }^{16} \mathrm{O}_{8}{ }^{32} \mathrm{~S}\right): 436.1192$, found: 436.119.

\section{(7RS)-7-Azido-1,2,3,10-tetramethoxy-6,7-dihydrobenzo[a]heptalen-9(5H)-one (rac-30)}

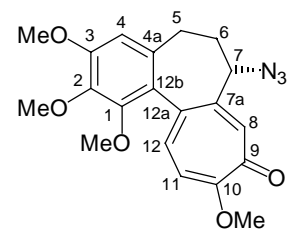

A solution of mesylether (rac-28) (180 mg, $0.41 \mathrm{mmol})$ and $\mathrm{NaN}_{3}(293 \mathrm{mg}, 11$ equiv.) in abs. DMSO $(20 \mathrm{~mL})$ was heated three days at $50{ }^{\circ} \mathrm{C}$. Water $(5 \mathrm{~mL})$ was then added and the solution was extracted with $\mathrm{CH}_{2} \mathrm{Cl}_{2}(3 \times 10 \mathrm{~mL})$. The combined organic extracts were then washed with saturated $\mathrm{NaHCO}_{3}(10 \mathrm{~mL})$ and brine $(10 \mathrm{~mL})$ and dried over $\mathrm{Na}_{2} \mathrm{SO}_{4}$. The raw product was purified by flash chromatography $\left(\mathrm{CH}_{2} \mathrm{Cl}_{2}\right.$ /acetone 1:1) to afford $122 \mathrm{mg}(77 \%)$ of a yellow oil.

$\mathbf{R}_{f}\left(\mathrm{SiO}_{2}, \mathrm{CHCl}_{3} / \mathrm{MeOH} 20: 1\right)=0.30$

IR (ATR): $\tilde{v}\left[\mathrm{~cm}^{-1}\right]=2936$ (s, br.), 2103 (ss, N $), 1614$ (m), 1585 (ss), 1486 (m), 1454 (m), 1431 (w), 1395 (m), 1346 (m), 1319 (m), 1248 (ss), 1137 (m), 1088 (m), 1064 (w), 1021 (m), $983(\mathrm{w}), 910(\mathrm{w}), 877(\mathrm{w}), 730(\mathrm{~m})$. 
${ }^{1}$ H-NMR $\left(300 \mathrm{MHz}, \mathrm{CDCl}_{3}\right): \delta[\mathrm{ppm}]=1.86(\mathrm{~m}, 1 \mathrm{H}), 2.40(\mathrm{~m}, 3 \mathrm{H}), 3.66(\mathrm{~s}, 3 \mathrm{H}, \mathrm{OMe}), 3.90$ (s, 6H, 2×OMe), $3.98(\mathrm{~s}, 3 \mathrm{H}, \mathrm{OMe}), 4.25(\mathrm{~m}, 1 \mathrm{H}, 7-\mathrm{H}), 6.52(\mathrm{~s}, 1 \mathrm{H}, 4-\mathrm{H}), 6.78\left(\mathrm{~d},{ }^{3} J=10.5\right.$ $\mathrm{Hz}, 1 \mathrm{H}), 7.19\left(\mathrm{~d},{ }^{3} J=10.5 \mathrm{~Hz}, 1 \mathrm{H}\right), 7.64$ (s, 1H, 8-H).

${ }^{13}$ C-NMR $\left(75 \mathrm{MHz}, \mathrm{CDCl}_{3}\right): \delta[\mathrm{ppm}]=29.75(\mathrm{t}, \mathrm{C}-5), 36.85(\mathrm{t}, \mathrm{C}-6), 56.05$ (q, OMe), 56.31 (q, OMe), 61.07 (q, OMe), 61.13(q, OMe), 62.98 (d, C-7), 107.18 (d, C-4), 111.68 (d, C-11), 120.46 (s), 121.10 (s), 132.64 (d, C-8), 134.03 (s), 134.89 (s), 135.02 (d, C-12), 147.32 (s), 153.66 (s), 164.11 (s, C-10), 179.48 (s, C-9).

MS (DIP-EI, $70 \mathrm{eV}): \mathrm{m} / \mathrm{z}(\%)=383(18)\left[\mathrm{M}^{+}\right], 355$ (12), 340 (100), 312 (43), 284 (81), 268 (23), 254 (12), 240 (15), 226 (12), 198 (10), 181 (40), 155 (13), 139 (17), 127 (17), 115 (14), 77 (12), 63 (12).

HRMS (EI): calc. for $[\mathrm{M}]]^{+}\left({ }^{12} \mathrm{C}_{20} \mathrm{H}_{21}{ }^{16} \mathrm{O}_{5}{ }^{14} \mathrm{~N}_{3}\right): 383.1481$, found: 383.148 .

\section{(7RS)-Colchicine (rac-1)}

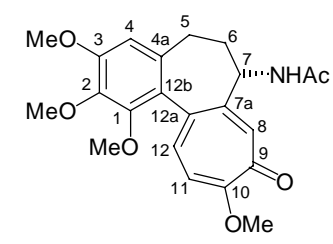

$\mathrm{Pd} / \mathrm{C}(10 \% \mathrm{wt})$ was added to a solution of azide ( $\mathrm{rac}-\mathbf{2 8})$ (48 $\mathrm{mg}, 0.12 \mathrm{mmol})$ in abs. EtOH (2 $\mathrm{mL}$ ). The resulting suspension was placed under hydrogen (1 bar) and stirred overnight. The suspension was then filtered over a short bed of Celite, the solvent was removed under vacuum to afford a brown oil. The raw product was purified by radial chromatography $\left(\mathrm{CH}_{2} \mathrm{Cl}_{2}\right.$ /acetone $\left.1: 1\right)$ to afford $35.7 \mathrm{mg}$ of a yellow powder (79\%) corresponding to the deacetylaminocolchicine.

$\mathbf{R}_{f}\left(\mathrm{SiO}_{2}, \mathrm{CHCl}_{3} / \mathrm{MeOH} 20: 1\right)=0.10$

IR (ATR): $\tilde{v}\left[\mathrm{~cm}^{-1}\right]=2946$ (s, br.), 1702 (m), 1590 (ss), 1488 (m), 1454 (m), 1401 (w), 1319 (m), 1247 (ss), 1137 (w), $1091(\mathrm{~m}), 983$ (w), 910 (w), 822 (w), 728 (m).

${ }^{1} \mathbf{H}-\mathbf{N M R}\left(250 \mathrm{MHz}, \mathrm{CDCl}_{3}\right): \delta[\mathrm{ppm}]=1.58(\mathrm{~m}, 1 \mathrm{H}), 2.32(\mathrm{~m}, 3 \mathrm{H}), 3.63$ (s, 3H, OMe), 3.69 (m, 1H, 7-H), 3.88 (s, 6H, 2×OMe), 3.97 (s, 3H, OMe), $6.51(\mathrm{~s}, 1 \mathrm{H}, 4-\mathrm{H}), 6.77$ (d, ${ }^{3} J=11$ $\mathrm{Hz}, 1 \mathrm{H}), 7.16\left(\mathrm{~d},{ }^{3} \mathrm{~J}=11 \mathrm{~Hz}, 1 \mathrm{H}\right), 7.71(\mathrm{~s}, 1 \mathrm{H}, 8-\mathrm{H})$.

${ }^{13}$ C-NMR (62.5 MHz, $\left.\mathrm{CDCl}_{3}\right): \delta[\mathrm{ppm}]=30.60(\mathrm{t}, \mathrm{C}-5), 40.40(\mathrm{t}, \mathrm{C}-6), 53.76$ (q, OMe), 56.04 (q, OMe), 56.22 (q, OMe), 61.01 (q, OMe), 84.77 (d, C-7), 106.98 (d, C-4), 111.73 (d, C-11), 131.71 (d, C-8), 134.47 (s), 136.37 (s), 141.15, 150.71 (s), 153.31 (s), 163.77 (s, C-10), 179.61 (s, C-9). 
MS (DIP-EI, $70 \mathrm{eV}): \mathrm{m} / z(\%)=357(86)\left[\mathrm{M}^{+}\right], 328$ (25), 312 (78), 298 (100), 281 (34), 254

(27), 239 (15), 207 (53), 181 (20), 165 (16), 152 (20), 141 (26), 115 (25), 77 (25).

HRMS (EI): calc. for $[\mathrm{M}]{ }^{+}\left({ }^{12} \mathrm{C}_{20} \mathrm{H}_{23}{ }^{16} \mathrm{O}_{5}{ }^{14} \mathrm{~N}\right): 357.401$, found: 357.157

To a solution of this product $(20 \mathrm{mg}, 0.056 \mathrm{mmol})$ in distilled pyridine $(2 \mathrm{~mL})$ was added freshly distilled acetanhydride $(0.21 \mathrm{~mL}, 2.23 \mathrm{mmol})$ under argon. After $10 \mathrm{~min}$, pyridine is evaporated under vaccum to give a brown oil. Purification by radial chromatography afforded $17.9 \mathrm{mg}$ of a pale yellow solid $(0.045 \mathrm{mmol})$. NMR data were compared to those of commercially available (-)-colchicine.

$\mathbf{R}_{f}\left(\mathrm{SiO}_{2}\right.$, acetone $)=0.25$

${ }^{1} \mathbf{H}-\mathbf{N M R}\left(300 \mathrm{MHz}, \mathrm{CDCl}_{3}\right): \delta[\mathrm{ppm}]=1.88\left(\mathrm{~s}, 3 \mathrm{H}, \mathrm{CH}_{3}-\mathrm{Ac}\right), 2.20(\mathrm{~m}, 3 \mathrm{H}), 2.46(\mathrm{~m}, 1 \mathrm{H})$, 3.58 (s, 3H, OMe), 3.83 (s, 3H, OMe), 3.87 (s, 3H, OMe), 3.94 (s, 3H, OMe), 4,57 (m, 1H, 7H), 6.47 (s, 1H, 4-H), $6.84\left(\mathrm{~d},{ }^{3} J=11 \mathrm{~Hz}, 1 \mathrm{H}\right), 7.29\left(\mathrm{~d},{ }^{3} J=11 \mathrm{~Hz}, 1 \mathrm{H}\right), 7.59$ (s, 1H, 8-H).

${ }^{13}$ C-NMR $\left(75 \mathrm{MHz}, \mathrm{CDCl}_{3}\right): \delta[\mathrm{ppm}]=22.49(\mathrm{q}, \mathrm{Ac}), 29.72(\mathrm{t}, \mathrm{C}-5), 36.07(\mathrm{t}, \mathrm{C}-6), 52.68$ (d, C-7), 55.96 (q, OMe), 56.30 (q, OMe), 61.20 (q, OMe), 61.40 (q, OMe), 107.14 (d, C-4), 112.90 (d, C-11), 125.40 (s), 130.18 (d, C-8), 134.15 (s), 135.56 (d, C-12), 136.94 (s), 141.40 (s), 150.94 (s), 152.76 (s), 153.36 (s), 163.83(s, C-10), 170.06 (s, CO-Ac), 179.32 (s, C-9). 


\section{Crystallographic data}

\section{(3R)-5-(2-Iodo-3,4,5-trimethoxyphenyl)-1-(trimethylsilyl)pent-1-yn-3-ol (8)}

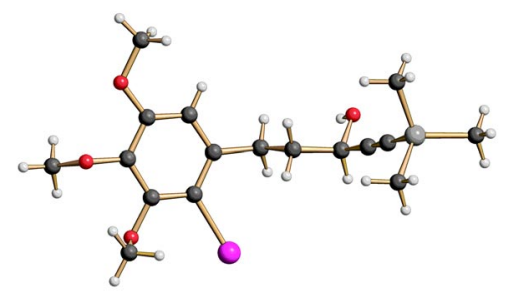

Table 1. Crystal data and structure refinement for $\mathbf{8}$.

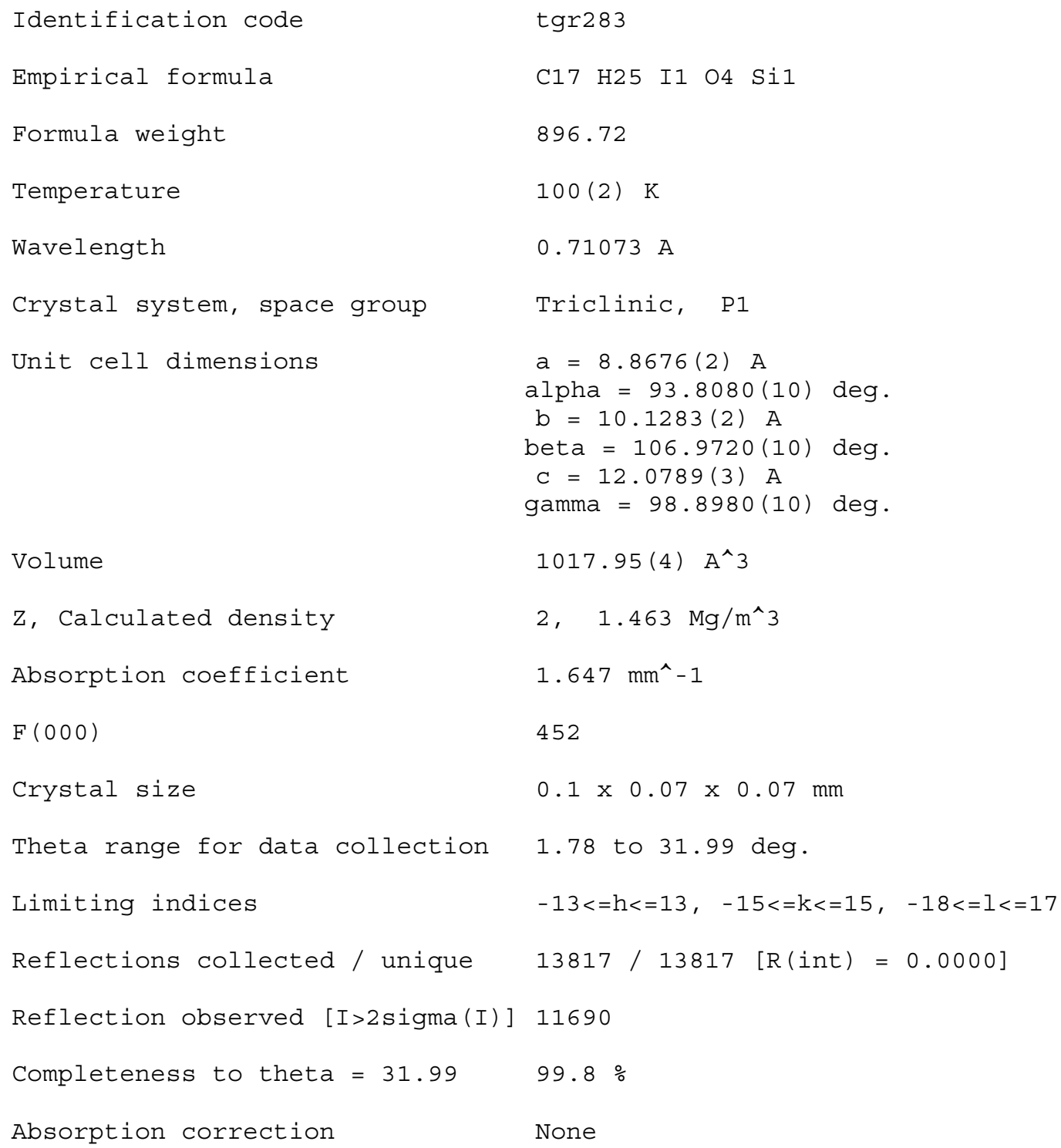




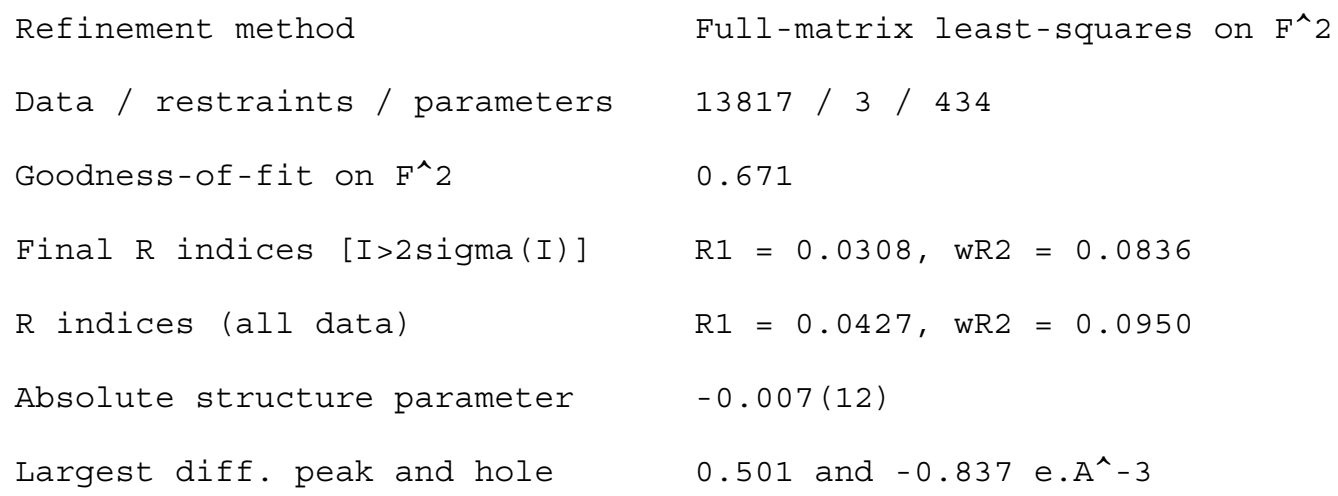

$(7 R, 9 R, 12 \mathrm{a} R)-7-[[$ tert-Butyl(dimethyl)silyl]oxy]-1,2,3-trimethoxy-9,12a-epoxy$6,7,9,11,12,12 a-h e x a h y d r o b e n z o[a]$ heptalen-10 $(5 H)$-one (12)

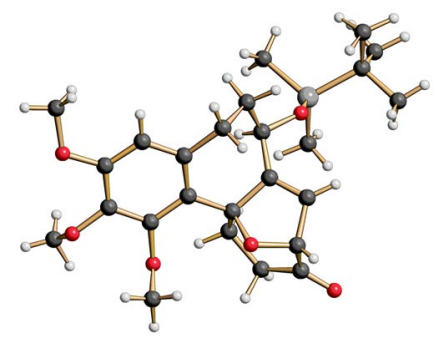

Table 2. Crystal data and structure refinement for $\mathbf{1 2}$.

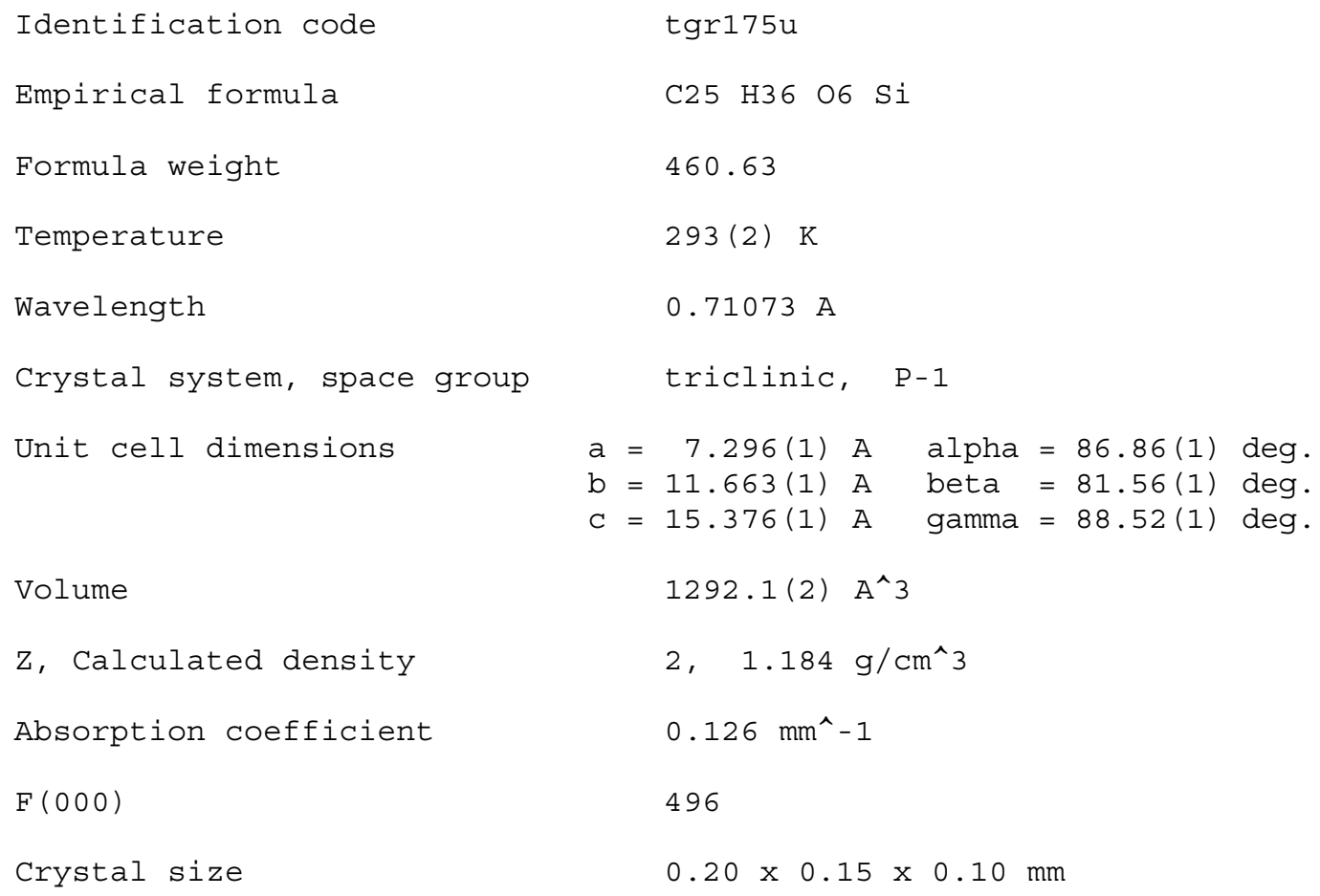




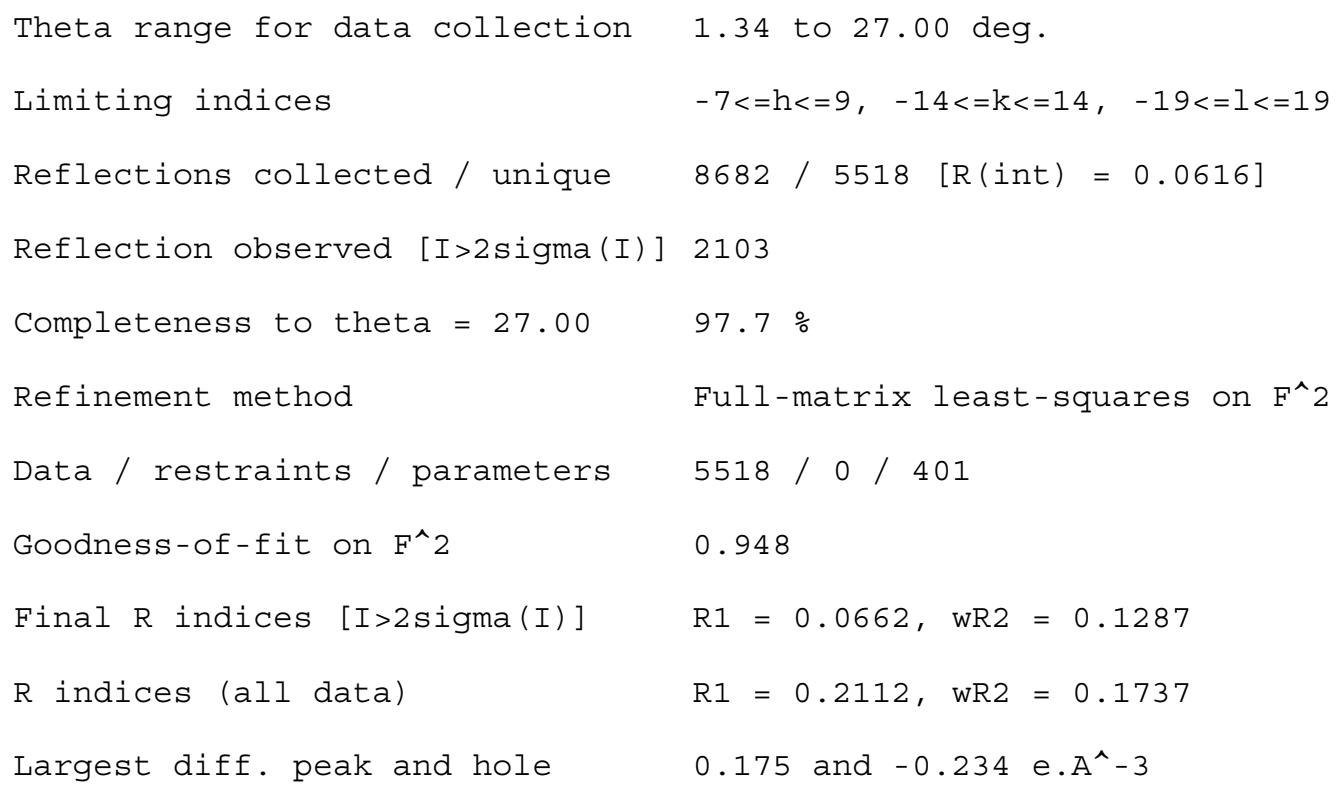

\section{(7R)-7-[[tert-Butyl(dimethyl)silyl]oxy]-1,2,3,9-tetramethoxy-6,7- dihydrobenzo $[a]$ heptalen-10(5H)-one $(25)$}

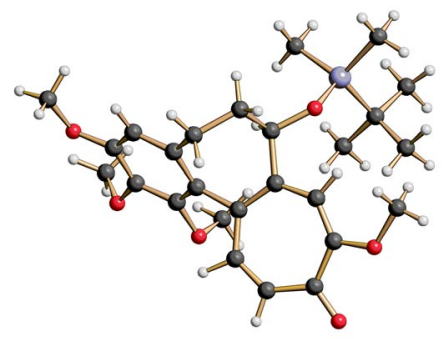

Table 3. Crystal data and structure refinement for 25.

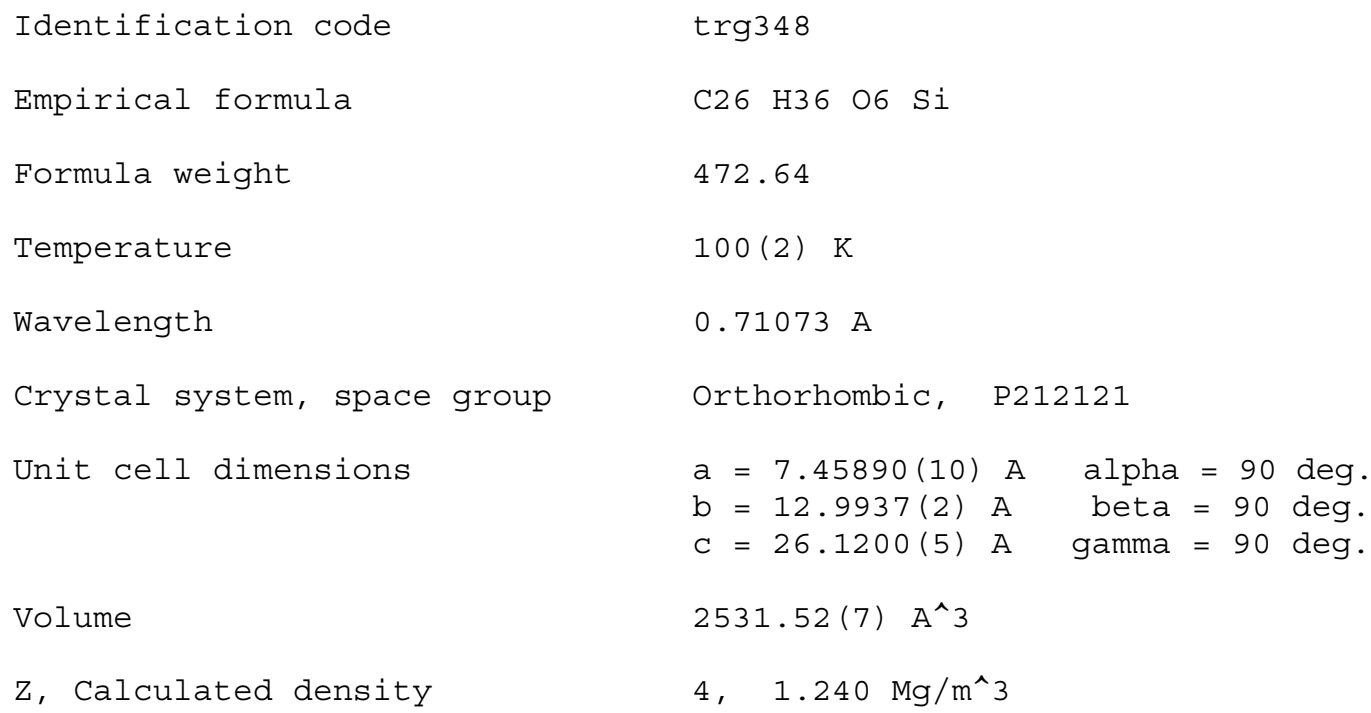




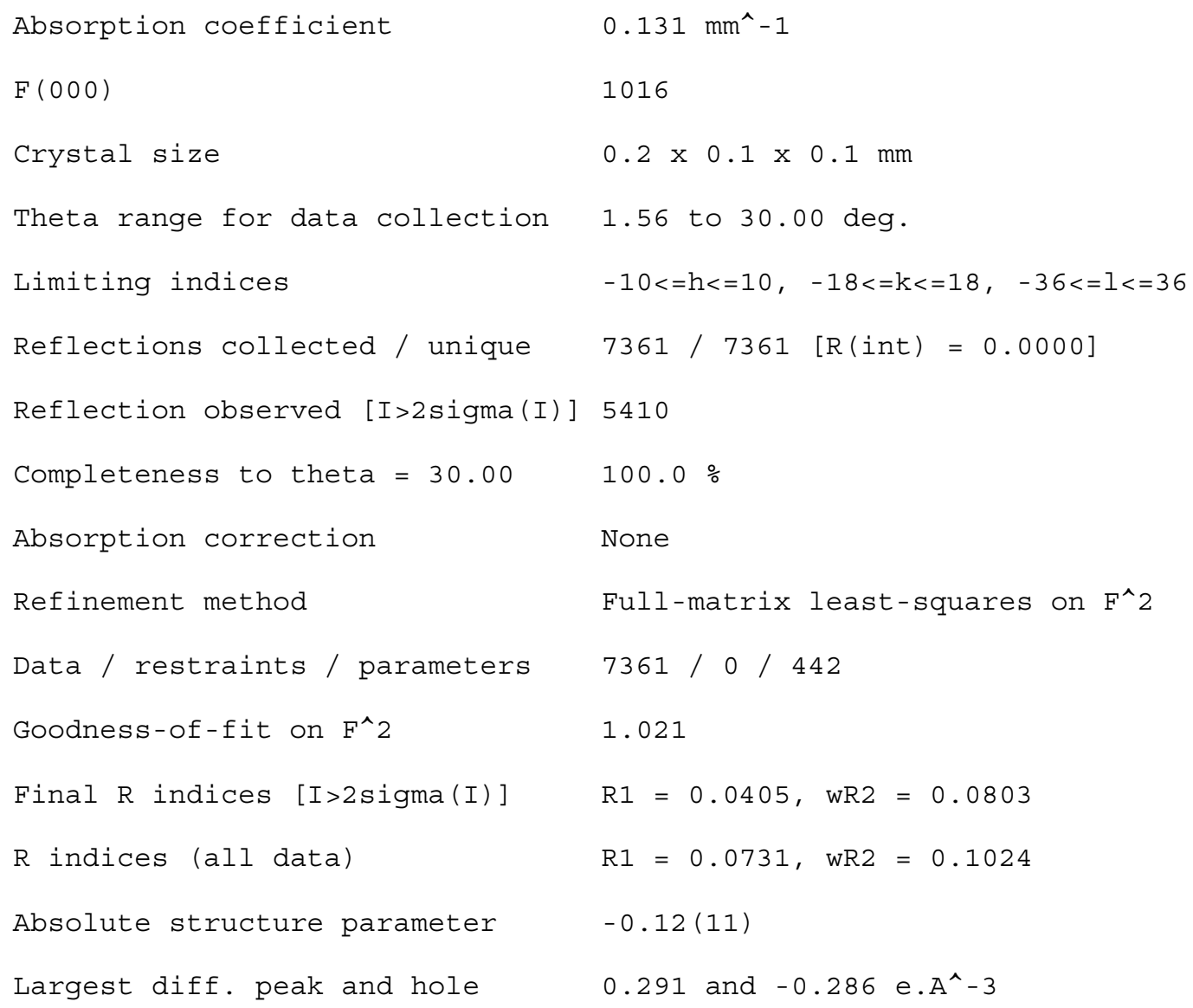

(7R)-7-Acetamino-1,2,3,10-tetramethoxy-6,7-dihydrobenzo[ $a]$ heptalen-9(5H)-one (32)

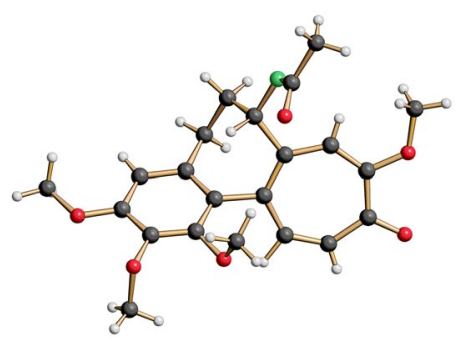

Table 4. Crystal data and structure refinement for $\mathbf{3 2}$.

$\begin{array}{ll}\text { Identification code } & \text { vb48 } \\ \text { Empirical formula } & \text { C32 H51 N O9 } \\ \text { Formula weight } & 593.74 \\ \text { Temperature } & 100(2) \mathrm{K} \\ \text { Wavelength } & 0.71073 \mathrm{~A}\end{array}$


Crystal system, space group

Unit cell dimensions

$73.381(5)$ deg.

Volume

Z, Calculated density

Absorption coefficient

F ( 000$)$

Crystal size

Theta range for data collection

Limiting indices

Reflections collected / unique

Reflection observed [I>2sigma(I)

Completeness to theta $=25.00$

Absorption correction

Refinement method

Data / restraints / parameters

Goodness-of-fit on $\mathrm{F}^{\wedge} 2$

Final $\mathrm{R}$ indices [I>2sigma(I)]

R indices (all data)

Absolute structure parameter

Largest diff. peak and hole
Monoclinic, P21

$\mathrm{a}=10.4972(19) \mathrm{A} \quad$ alpha $=90 \mathrm{deg}$.

$\mathrm{b}=12.7999(14) \mathrm{A}$ beta $=$

$\mathrm{C}=13.099(2) \mathrm{A}$ gamma $=90 \mathrm{deg}$.

$1686.5(5) \mathrm{A}^{\wedge} 3$

2, $1.169 \mathrm{Mg} / \mathrm{m}^{\wedge} 3$

$0.084 \mathrm{~mm}^{\wedge}-1$

644

$0.3 \times 0.2 \times 0.05 \mathrm{~mm}$

2.02 to 25.00 deg.

$-12<=\mathrm{h}<=12, \quad-11<=\mathrm{k}<=15, \quad-13<=1<=15$

$7065 / 3080[R($ int $)=0.0786]$

1593

$98.7 \%$

None

Full-matrix least-squares on $\mathrm{F}^{\wedge} 2$

$3080 / 1 / 404$

1.011

$\mathrm{R} 1=0.0818, \mathrm{wR} 2=0.1871$

$\mathrm{R} 1=0.1691, \mathrm{wR} 2=0.2284$

$0(3)$

0.405 and -0.315 e.A^-3 